\title{
POLARIZATION DEPENDENCE OF MICROWAVE "IONIZATION" OF EXCITED HYDROGEN ATOMS
}

\author{
P.M. KOCH* \\ Department of Physics and Astronomy, State University of New York \\ Stony Brook, NY 11794-3800, USA
}

\begin{abstract}
After a brief review of the ionization of excited hydrogen atoms by a linearly polarized field, we discuss experimental results for the polarization dependence of this process. Experiments at $\omega / 2 \pi=9.904 \mathrm{GHz}$ used two different ranges of principal quantum number $n_{0}$ between 29 and 98 . At low scaled frequencies, $\Omega_{0} \lesssim 0.1$, ionization data for certain narrow ranges of $n_{0}$ exhibit striking sensitivity to fields with elliptical polarization not too far from circular polarization. Classical calculations reproduce this behavior and show it to be the result of $2 \omega$ driving terms that appear when the Hamiltonian is transformed to a frame rotating at $\omega$. It shows how higher-dimensional dynamics can influence the ionization and be used to control it when the polarization departs from linear or circular polarization. At higher scaled frequencies, $0.6 \lesssim \Omega_{0} \lesssim 1.4$, near the onset of ionization circularly and elliptically polarized data show surprising similarities with linearly polarized data in a parameter regime where the ionization dynamics is dominated by the influence of the pendulumlike resonance zone centered at scaled frequency $n_{0}^{3} \omega \equiv \Omega_{0}=1$. The stabilizing influence of this zone can be understood classically, but nonclassical stability associated with quantal separatrix states at its edge is a semiclassical effect.
\end{abstract}

PACS numbers: $32.80 . \mathrm{Rm}, 05.45 .+\mathrm{b}, 42.50 . \mathrm{Hz}$

\section{Introduction}

Experiments on the ionization of hydrogen atoms with principal quantum number $n_{0}$ by a linearly polarized (LP) microwave electric field $F \sin (\omega t)$ have been a cornucopia of data. For recent reviews and commentary that emphasize experimental data obtained at Stony Brook and compare them to other experimental data and classical, semiclassical, and quantal calculations, see [1, 2]; for ones appearing earlier in the 1990s, see [3-8]. Reviews or extended articles in the 1990 s that emphasize theoretical calculations, but contain comparisons with or commentary on LP ionization data, include [9-29].

*Internet: Peter.Koch@sunysb.edu. 
From the vantage point of semiclassical theory, the experiments attract attention because for a wide range of parameters the onset of ionization coincides classically with the onset of irregular (chaotic) trajectories. This system is the paradigm for the experimental/theoretical/numerical study of non-perturbative quantal dynamics in a time-dependent, low-dimensional, classically chaotic system. All previous LP experiments, as well as those in this paper for varying polarization, required the (net) absorption of many photons to reach the ionization continuum. This, and so many quantal states being coupled by the strong driving field, renders quantal perturbation theory questionable, if not useless, as a calculational method for simulating the experiments. Brute-force numerical integrations of the Schrödinger equation on large, fast computers are used, but often the crucial insights into the complexities of the ionization dynamics have come from semiclassical or even fully classical theory and calculations.

Dynamics is about time scales, which means comparing frequencies. Because of its special dynamical symmetries, the classical bound orbits of the unperturbed, non-relativistic 3d hydrogen atom at total energy $E<0$ depend only upon the principal action $I_{0}$. In atomic units (a.u. $)^{\dagger}$ classically $E=-\left(2 I_{0}^{2}\right)^{-1}$; quantally, replace $I_{0}$ with $n_{0}$. Therefore, at $E<0$ there is only the Kepler frequency $\omega_{\mathrm{K}}=\partial E / \partial I_{0}=I_{0}^{-3}$. Exemplifying Bohr's correspondence principle, in the limit of $n_{0} \rightarrow \infty, \omega_{\mathrm{K}}$ is the frequency splitting between adjacent $n$-states, $|\Delta n|=1$. For large but finite $n_{0}, \omega_{\mathrm{K}}$ is close to the average of the $\Delta n= \pm 1$ splittings.

When the excited hydrogen atom is driven at frequency $\omega$, the important frequency ratio is the scaled frequency $\omega / \omega_{\mathrm{K}}=n_{0}^{3} \omega \equiv \Omega_{0}$. Similarly, the scaled amplitude $n_{0}^{4} F \equiv F_{0}$ is the ratio of the peak amplitude of the driving field to the Coulomb field at the Bohr (circular) orbit $r_{n}=n^{2}$ a.u. As was first shown in [31] the resultant classical dynamics does not depend independently on $\omega, F$, and $I_{0}$; it depends on the ratios $\Omega_{0}$ and $F_{0}$. However, a non-zero Planck's constant $\hbar$ spoils the classical scaling invariance in the quantal dynamics. Varying $n_{0}, \omega$, and $F$ in such a way as to keep $\Omega_{0}$ and $F_{0}$ constant changes the size of the effective $\hbar, \widetilde{\hbar}=\hbar / n_{0}$. However, Refs. [1, 2, 32, 33], as well as the discussion in Sec. 5 below, show that the experimental, i.e., quantal, ionization data can follow classical scaling for at least up to $50 \%$ changes in $\widetilde{\hbar}$.

All results mentioned above were obtained for the case of an LP driving field.

It is well known from pulsed laser experiments with tightly bound atoms that the polarization of an intense electromagnetic field can strongly influence atomic ionization when many photons must be absorbed. In a perturbation expansion polarization-dependent electric dipole selection rules determine pathways through unperturbed states, so small changes in polarization may dramatically vary the ionization rate for fixed peak field amplitude. Except for the case [34] of few-photon $(\lesssim 3$ ) ionization dominated by a resonance between intermediate bound states, early optical [35] and microwave [36] experiments, using atoms in initial states

\footnotetext{
${ }^{\dagger}$ Atomic units (a.u.) are defined by setting $(2 \pi)^{-1}$.Planck's constant, $\hbar$, the electron mass, $m_{\mathrm{e}}$, the antielectron charge, $e$, and the electrostatic constant, $4 \pi \varepsilon_{0}$, all equal to one. Definitions and numerical values for a.u. are listed in Ref. [30]. When confronting experimental data obtained with real hydrogen atoms or its isotopes, one should use physical a.u. that result from substituting for $m_{\mathbf{e}}$ the reduced electron mass $\mu_{\mathbf{e}}$.
} 
$\left|n_{0}, \ell, m\right\rangle$ with low values of the angular momentum $(\ell \leq 2)$ and a driving frequency $\omega \ll\left(E_{n_{0}+1}-E_{n_{0}}\right) / \hbar$, showed that many-photon $(\gtrsim 11)$ ionization in a circularly polarized (CP) field required significantly larger field intensities than in an LP field. A smaller effective quantal density of participating, unperturbed states in $\mathrm{CP}$ fields was used to explain [37] these many-photon results. The monotonic change of the azimuthal quantum number $m$ with each absorbed photon forces an eventual monotonic increase in $\ell$, whereas in LP the $\Delta m=0$ selection rule does not seriously constrain the upward and downward movement in $\ell$ permitted by the $\Delta \ell= \pm 1$ selection rule. Classically, quasistatic pictures including effects of frame rotation and an angular momentum barrier were used $[38,39]$ to explain the microwave ionization results. Nevertheless, these explanations are all "rules of thumb", applicable only to particular cases. They have no universal validity. In a succinct description of our limited knowledge a recent review [40] stated, "the matter of polarization ... is more complicated than previously realized".

CP ionization experiments with pulsed lasers (for references, see above) and tightly bound atoms or with microwave fields and alkali Rydberg atoms $[36,38,41,42]$, as well as most theories to date, have focused on fields with $\Omega_{0}<1$. When $\Omega_{0} \ll 1$, a quasistatic view should be applicable if narrow quantal resonances [43] are avoided, while at higher frequencies dynamics should play an important role.

Early work on the non-LP ionization of excited hydrogen atoms was theoretical $[44,45]$, using classical analysis and numerical simulations to follow trajectories near $\Omega_{0}=1$ for the case of a CP ficld. The approximate classical resonance analysis presented in Sec. 6.F of [46] led to a conclusion that the onset of ionization for hydrogen atoms driven by a CP field near $\Omega_{0}=1$ would occur at a peak amplitude estimated to be 1.5-2 times below that for an LP field. This estimate correctly anticipated the trend found experimentally; see Sec. 5 below.

The recent flurry of theoretical activity on the ionization of Rydberg atoms by a CP field was stimulated by the first CP experiment with alkali atoms [36]. Since then several theoretical groups have published growing series of papers on excited hydrogen atoms driven by a strong CP field. These include [13, 39, 47-79]. Several contain the lively give-and-take of theoretical controversies not yet completely resolved, both about the ionization mechanism in a CP field and the existence and nature of nonspreading wave-packet states of hydrogen atoms in a strong CP field.

Unlike the LP and CP cases, the elliptically polarized (EP) case has no integrals of the motion: it has three degrees of freedom and is not conservative. Moreover, the case that interests us here, that of many unperturbed states being strongly coupled, is unlikely to be amenable to any simple selection rule analysis for LP vs. CP vs. EP driving.

Intended to be a reasonably self-contained, mid-length review that emphasizes experimental and theoretical work on hydrogen atoms, this paper is organized as follows. Section 2 describes the experimental method. Section 3 reviews what has been learned from previous LP experiments and theory. It describes six regimes of strong-field dynamical behavior, each lying within a certain range of the scaled frequency $\Omega_{0}$, though the dividing lines are fuzzy. Section 4 presents experimen- 
tal and classical theoretical results for the polarization dependence of ionization at low scaled frequencies, $\Omega_{0} \lesssim 0.1$. For certain, narrow ranges of $n_{0}$, striking EP-induced resonances dominate the ionization dynamics. Section 5 presents experimental and classical theoretical results for the polarization dependence of ionization for $0.6 \lesssim \Omega_{0} \lesssim 1.4$. This includes a range where the ionization dynamics is dominated by the influence of the main, pendulumlike resonance zone centered at $\Omega_{0}=1$. Section 6 presents some conclusions and directions for future work.

\section{Experimental method}

\subsection{Apparatus}

Because the details of the experimental method are given in $[1,2,80-83]$, this discussion is brief. Ions extracted from a hydrogen ion source were accelerated, focused, and deflected in a mass-analyzer magnet tuned to transmit protons. $14.6 \mathrm{keV} \mathrm{H} \mathrm{H}^{+} \mathrm{Xe}$ electron-transfer collisions produced fast hydrogen atoms with an approximately $n^{-3}$-weighted distribution of states. A static electric field $\gtrsim 100 \mathrm{kV} / \mathrm{cm}$ ionized all $\mathrm{H}(n>9)$ atoms, while those with $n \lesssim 6$ radiatively decayed in flight. With parabolic quantum numbers $\left(n, n_{1},|m|\right)$ labeling Stark substates, in a $29.2 \mathrm{kV} / \mathrm{cm}$ static field half of the $(7,0,0)$ population was driven into $(10,0,0)$ by a $\mathrm{CO}_{2}$ laser. Another $\mathrm{CO}_{2}$ laser drove the transition $(10,0,0) \rightarrow$ $\left(n_{0}, 0,0\right)$ in a static field whose strength was between a few to a few hundred $\mathrm{V} / \mathrm{cm}$. This created a beam of $\mathrm{H}\left(n_{0}\right)$ atoms that was collimated by a $0.21 \mathrm{~cm}$ diameter aperture before it traversed the $9.904 \mathrm{GHz}$ cavity described in Sec. 2.2. This paper involves $\mathrm{H}\left(n_{0}\right)$ atoms with individual $n_{0}$-values between 29 and 98 . A surviving atom signal $I_{\text {surv }}$ consisting of energy-labeled protons was produced by ionization of excited atoms in a voltage-labeled, rectilinear, $9.8 \mathrm{GHz}$ cavity, followed by electrostatic deflection, transmission through an electrostatic filter lens, and detection in a particle multiplier; see Sec. $13.3 \mathrm{c}$ of [80]. A uniform, longitudinal $3.8 \mathrm{~V} / \mathrm{cm}$ field before the $9.8 \mathrm{GHz}$ cavity caused only atoms with $n$-values below the $n$ cutoff $n_{\mathrm{c}}^{\mathrm{q}} \approx 110$ to contribute to $I_{\text {surv }}$; see Sec. 2.3.3 of [1].

Reduction of the signal $I_{\text {surv }}$ was caused by $\mathrm{H}\left(n_{0}\right)$ in the $9.904 \mathrm{GHz}$ cavity being either truly ionized or excited to final $n$-values $n>n_{\mathrm{c}}^{\mathrm{q}}$. Calling these two contributions to the reduction in $I_{\text {surv }}$ an "ionization" signal [1], the "ionization" and survival probabilities are related as $P$ "ion" $=1-P_{\text {surv }}$. Experimentally, we measured $P_{\text {surv }}$, but we interpret this in terms of $P_{\text {"ion }}$; ; see Sec. 2.3.4 of [1]. Energetically, "ionization" required the net absorption of at least $\mathcal{N}=$ $1+\left[\left(\left(2 n_{0}^{2}\right)^{-1}-\left(2 n_{\mathrm{c}}^{\mathrm{q}^{2}}\right)^{-1}\right) / \omega\right]$ photons, where [...] means "the integer part of". At $\omega / 2 \pi=9.904 \mathrm{GHz}$, and taking $n_{\mathrm{c}}^{\mathrm{q}}=110, \mathcal{N}=396$ at $n_{0}=29$, dropping to $\mathcal{N}=8$ at $n_{0}=98$.

For clarity in the remainder of this paper, we no longer write "ionization" and "ion", but the presence of quotation marks will be understood.

\subsection{Microwave cavity}

The cylindrical brass cavity had inner dimensions length $L=2.57 \mathrm{~cm}$ and diameter $D=6.350 \mathrm{~cm}$. The beam traversed $0.26 \mathrm{~cm}$ diameter holes in the 
$0.159 \mathrm{~cm}$ thick entrance and $0.476 \mathrm{~cm}$ thick exit endcaps, respectively. Exciting the cavity via two spatially orthogonal coupling slots in the entrance endcap to resonate degenerate $\mathrm{TE}_{121}$ modes, one could vary at will the polarization of the $9.904 \mathrm{GHz}$ cavity field. The on-axis field was

$$
\boldsymbol{F}(t)=\lambda(t) \mathcal{F}[\widehat{\boldsymbol{X}} \alpha \sin (\omega t)+\widehat{\boldsymbol{Y}} \sin (\omega t+\delta)]
$$

with $0 \leq \alpha \leq 1$ and $0 \leq \delta \leq \frac{\pi}{2} ; 0 \leq \lambda(t) \leq 1$ is the 152 cycle, half-sine pulse envelope seen by the atoms in their rest frame, and $(X, Y, Z)$ are spatial coordinates in the laboratory frame. The ratio $F / \mathcal{F}$ of the peak amplitude $F$ to the amplitude $\mathcal{F}$ of the $\hat{\boldsymbol{Y}}$-component depends on the polarization. With an attenuator in one arm, to control $\alpha$, and a phase shifter in the other, to control $\delta$, we used the atoms to fine-tune the polarization. Extinguishing the power in one arm $(\alpha \leq 0.0002)$ created LP. To create EP one could either (i) keep $\alpha=1$ and vary $\delta$ or (ii) make $\alpha<1$ and keep $\delta=\pi / 2$ or (iii) vary both $\alpha$ and $\delta$. Experimentally it was more precise to use scheme (ii), but for comparisons with theory to be made below, it will be easier to characterize the EP via scheme (i), for which $F / \mathcal{F}=\sqrt{2} \cos (\delta / 2)$ varies from 1 , at $C P$, to $\sqrt{2}$, at $L P$.

For converting scalar microwa ve power measurements to $F$ (or $\mathcal{F}$ ) with an estimated $5 \%$ accuracy for the case of arbitrary polarization, we adapted the method presented for LP cavity fields in [84]. S. Zelazny [85] kindly checked this method for determining the power-to-amplitude calibration for our CP microwave ionization data for hydrogen. He used the same apparatus to take CP Stueckelberg oscillation data (for the case of LP fields, see [86]) for $\left(1 s n_{0} s\right)^{3} S_{1}$ He Rydberg atoms with $n_{0}$ near 30 . He calculated Floquet eigenvalues for He triplet Rydberg atoms in the CP field to provide quasienergy potential curves for modeling the experimental data and determining the absolute field amplitude to $5 \%$.

\subsection{Ionization curves}

Taken for LP, CP, and two values of EP, Fig. 1 shows representative ionization curves, $P_{\text {ion }}$ vs. the scaled peak field amplitude $F_{0}$, for two different, nearby $n_{0}$ values. For both the scaled frequency $\Omega_{0}$ is low enough to be in regime-II, as is described for LP in the next section. Little change occurs near the onset of ionization as the polarization is varied. However, this is not the case well past the onset of ionization: the CP curves "stretch out", reaching $P_{\text {ion }}=1$ only at a significantly larger value of $F_{0}$ than do the other curves. Why this happens, as well as what produces the structure evident in the $\delta=0.45 \pi \mathrm{EP}$ curve for $n_{0}=42$, will be explained in Sec. 4. For now we emphasize the usefulness of the stretching out for experimentally setting CP precisely. In fact, for large $P_{\text {ion }}$, say $P_{\text {ion }}=0.8$, the measured CP peak field thresholds were systematically larger than those for LP driving for $n_{0}=30, \ldots, 78$. (No similar, simple statement applies to smaller values of $P_{\text {ion }}$ and/or $75<n_{0} \leq 98$.) Because, for these parameters, small departures from CP to EP lowered the large- $P_{\text {ion }}$ threshold, we used this dependence, usually for $n_{0}=58$, to fine-tune the polarization to CP $[36,82,83]$ giving $\alpha=1.00 \pm 0.05$ and $\delta=90^{\circ} \pm 1^{\circ}$. 


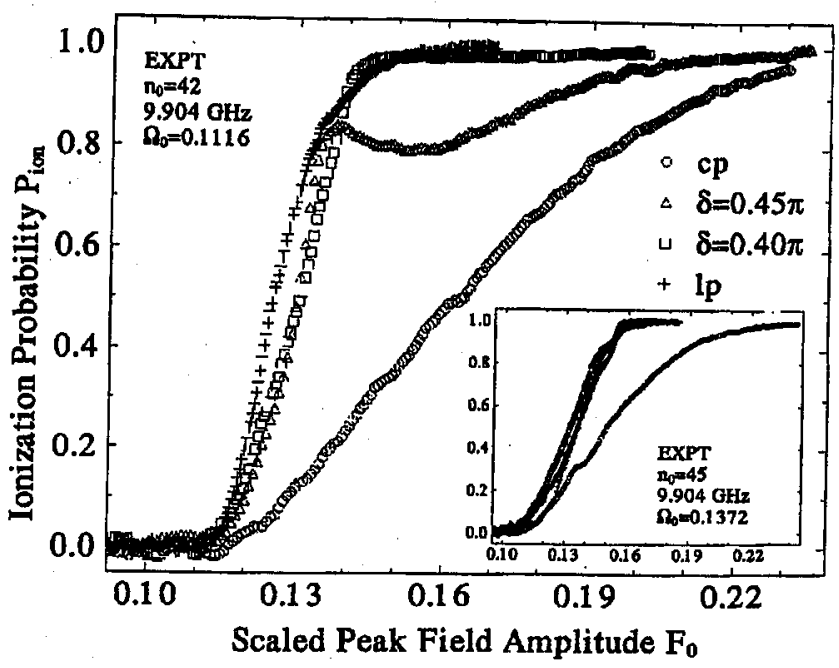

Fig. 1. A survey of the polarization dependence for experimental ionization curves for $n_{0}=42$ and $n_{0}=45$ (inset).

\section{Ionization by a linearly polarized field 3.1. Regimes of behavior}

In LP experiments at Stony Brook we have thus far varied $\Omega_{0}$ in a stepwise manner between $0.021-2.8$ with use of cavities between 7.6 and $36 \mathrm{GHz}$ and various ranges of $n_{0}$-values between 24 and 98 ; for reviews, see [1, 2, 6, 81]. We have also used a broadband, waveguide interaction region for a smaller number of LP studies between $26-40 \mathrm{GHz}$; see, e.g., [87-89] and see the early 9.6-11.4 GHz LP waveguide data in [90], reconsidered in Appendix B of [1]. The combined experimental-theoretical investigation of this wide dynamic range of $\Omega_{0}$ led to. classifying six different regimes of dynamical behavior, the first five of which we have studied experimentally. References $[1,2]$ discuss them in some detail. The dividing lines between these regimes are fuzzy.

Figure 2 is helpful for a brief description of regimes I through V. It compares two sets of our experimental data with the results of $3 \mathrm{~d}$ classical Monte Carlo calculations (3dCL) [91-93] that closely modeled the experimental situation: for each initial classical principal action $I_{0}$ a microcanonical distribution of orbits, which corresponds to the uniform distribution of quantal substates of fixed $n_{0}$ that entered the microwave cavity in the experiment [1]; the pulse envelope $\lambda(t)$; and the $n$ cutoff $n$. Plotted are scaled $10 \%$-thresholds, i.e., the scaled amplitude $n_{0}^{4} F(0.1)=F_{0}(0.1)$ at which $P_{\text {ion }}$ reaches 0.1 , vs. $\Omega_{0}$.

Regime-I: the quasistatic tunneling regime. When $\Omega_{0}$ gets low enough, one expects quantal penetration of the slowly oscillating Coulomb-Stark potential barrier to become the dominant ionization mechanism. Comparisons of $9.91 \mathrm{GHz}$ data for $n_{0}=24-32$ with $3 \mathrm{~d}$ quantal and theoretical calculations [94], reviewed in [1], show this behavior: as $n_{0}$ decreases with fixed $\omega$, the experimental onset of ionization falls systematically below the classical onset. A 1d theoretical 


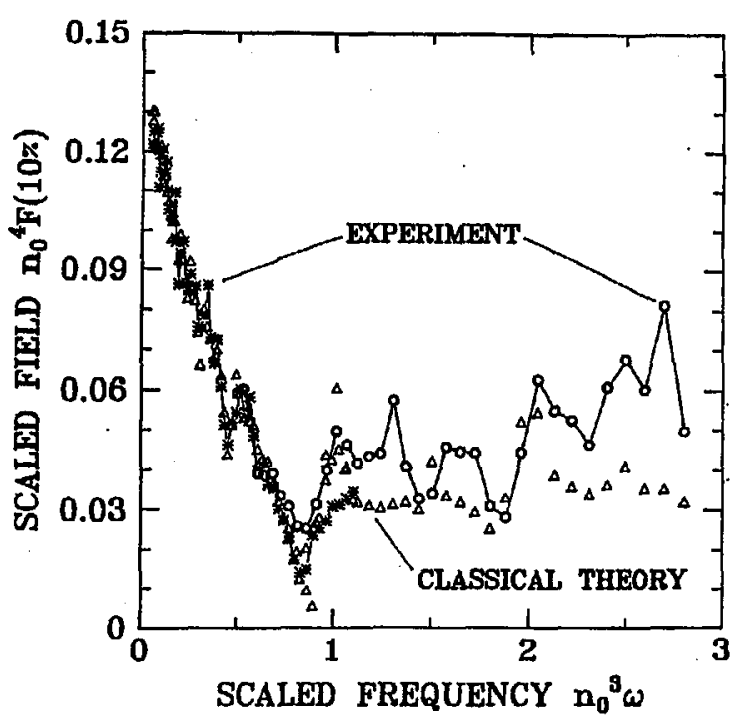

Fig. 2. Scaled amplitudes for $10 \%$ ionization probability for $3 \mathrm{~d}$ hydrogen atoms in an LP field. (*): $9.92 \mathrm{GHz}$ experiments with a flat-top pulse envelope with each $n_{0}=32, \ldots, 90$ and an $n$-cutoff $n_{c} \approx 92 ;(O): 36.02 \mathrm{GHz}$ experiment with a flat-top pulse envelope with each $n_{0}=45, \ldots, 80$ and an $n$-cutoff $n_{0} \approx 89 ;(\triangle): 3 \mathrm{~d}$ classical Monte-Carlo simulations of the experiments.

model $[43,95,96]$ reproduces and explains this behavior when the coupling constant $C_{n_{0}}=1.5 n_{0}\left(n_{0}^{3} \omega\right)\left(n_{0}^{4} F\right)$ between adjacent quantal adiabatic basis states is sufficiently small. At $9.91 \mathrm{GHz}$, this occurs for $n_{0} \lesssim 28$, or $\Omega_{0} \lesssim 0.03$; see Fig. 16 of [1]. Reference [43] predicts that the quantal resonances between adiabatic states, which are the distinctive feature of regime-II discussed next, will also be present in regime-I, but that as a function of frequency the resonances get exponentially narrow as $\Omega_{0}$ decreases.

Regime-II: the low frequency regime. For $0.05 \lesssim \Omega_{0} \lesssim 0.3$, some experimental microwave ionization and quench curves have shown structures such as non-monotonic bumps, steps, or changes in slope; see ionization curves in $[1,96]$ for examples. These structures can be understood as the consequence of amplitude-tuned resonances between the adiabatic basis states mentioned above. Other 1d quantal theoretical treatments [10, 97, 98] based in part on Floquet theory have given good numerical estimates for the onset of experimental ionization. In these treatments the experimental structures are caused by interactions at avoided crossings of quasienergy (Floquet) states.

Regime-III: the near-classical regime. For $\Omega_{0}$ between about $0.1-1.2$, the general agreement in Fig. 2 between experimental 10\%-thresholds and those obtained from $3 \mathrm{dCL}$ must mean that the strong driving field is coupling not a small number of quantal states: the density of participating quantal states must be high enough for near-classical behavior. The onset of classical ionization coincides with the onset of unstable (chaotic) classical trajectories; see [1,2] for lists of references. The 
classical dynamics in regime-III and neighboring regimes is strongly influenced by nonlinear (trapping) resonances, wherein the local motion is pendulumlike; see previously cited references and $[1,2,19,28,99-105]$. They give enhanced stability, i.e., higher ionization thresholds, when $\Omega_{0}$ is near simple rational fractions and give a semiclassical explanation for the bumps in the experimental data in Fig. 2 for $\Omega_{0}$ near, e.g., $1 / 3,1 / 2,1$, and 2. Nevertheless, localized disagreement in Fig. 2 shows that quantal effects are still important in regime-III.

Notice in Fig. 2 the good agreement for $\Omega_{0}$ between about $0.5-0.8$ for the experimental data sets taken at two different frequencies, 9.9 and $36 \mathrm{GHz}$, for two different ranges of $n_{0}$. This is an example of classical scaling in the quantal dynamics. As is explained in $[1,2]$, that the $9.9 \mathrm{GHz}$ data lie systematically below the $36 \mathrm{GHz}$ data for $\Omega_{0}$ between $0.8-1.1$ is an $n$-cutoff effect. This occurs when initial $n_{0}$ values in one data set are much closer to the $n$ cutoff than in the other data set. See also Sec. 3.3 and Fig. 4, below.

Regime-IV: the transition regime. In the range $1 \lesssim \Omega_{0} \lesssim 2$, Fig. 2 shows that the experimental data begin to deviate in a systematic way from $3 \mathrm{dCL}$, although similarities remain near resonances, e.g., near $\Omega_{0}=1$ and 2. But notice, in particular, that the large measured $[1,2,33,87,89,106,107]$ peak near $\Omega_{0}=1.3$ is absent classically; we shall return to this in Sec. 5 .

The first data [106] displaying this striking example of nonclassical local stability with $3 \mathrm{~d}$ atoms stimulated 1d quantal calculations [108] that confirmed the effect via numerical integrations of the time-dependent Schrödinger equation and gave a clear semiclassical physical picture $[19,109]$ for it. Via projections of the Husimi (coherent-state-smoothed Wigner function) wave function distribution onto phase portraits of the periodically driven $1 \mathrm{~d}$ classical hydrogen atom, the stability was shown to be associated with a wave packet localized in the chaotic part of the phase space just outside the main pendulumlike resonance zone, i.e., the one centered at $\Omega_{0}=1$. The preparation of this wave packet was found numerically [108-110] to be associated with the slow turn-on of the driving field amplitude by the pulse envelope $\lambda(t)$. Moreover, subsequent $1 \mathrm{~d}$ theoretical work [22, 111] associated this nonclassical, local stability specifically with wave packet states living near the separatrix of the pendulumlike classical motion. For earlier work on separatrix states in the kicked rotor model, see [112-114].

Following earlier quantal theoretical work carried out in (2 or 3$) \mathrm{d}+$ time [115-118], recent quantal calculations using the complex-coordinate-rotated Floquet method [11-13, 17, 49, 119-122] have extended to $3 \mathrm{~d}$ the treatment of hydrogen in LP microwaves. However, the $3 \mathrm{~d}$ Floquet calculations still have to address the important role of the slow experimental turn-on $\lambda(t)$ in the $3 \mathrm{~d}$ experiments.

References [33, 107] used a powerful experimental technique to demonstrate the classical "origin" of the nonclassical local stability peak at $\Omega_{0} \approx 1.3$, viz., that it scaled classically; see also $[1,2,32]$. When $n_{0}, \omega$, and the microwave amplitude $F$ were all varied, the nonclassical local stability remained near $\Omega_{0}=1.3$ and for a nearly constant value of the scaled $10 \%$-threshold $F_{0}(0.1)$. This was shown for three ranges of $n_{0}$ at the driving frequencies $26.4,30.36$, and $36.0 \mathrm{GHz}$.

Moreover, Refs. [2, 87, 89] showed experimentally and theoretically that broad-band noise added to the coherent driving field had a strong effect on the 
nonclassical peaks. Indeed, the addition of noise was used as an experimental tool for finding the influence of separatrix states both below and above the resonance zone centered at $\Omega_{0}=1$. Reference [89] demonstrated a strong frequency dependence near these nonclassical peaks that was also strongly affected by broad-band noise. Remarkably, both the frequency dependence and the influence of added noise observed experimentally with $3 \mathrm{~d}$ hydrogen atoms near the onset of ionization was reproduced with $1 \mathrm{~d}$ quantal numerical simulations based on an integration of the time-dependent Schrödinger equation.

Section 5 presents experimental evidence for the persistence with varying polarization of the observed nonclassical local stability associated with separatrix states.

Regime-V: the high-frequency regime. When $\Omega_{0}>2$, observed threshold amplitudes in Fig. 2 rise systematically above classical thresholds. The LP experiments in [106] were stimulated by and provided the first experimental confirmation of the earlier prediction of this quantal effect for periodically driven hydrogen atoms $[115,116,123,124]$; see also $[11,19,97,119]$ and the reviews $[117,125-128]$. Subsequent microwave ionization experiments with hydrogen [129] and rubidium [130] Rydberg atoms confirmed the rise of the thresholds.

The theoretical prediction of the quantal stabilizing effect of dynamical localization followed from the hydrogen atom periodically driven by a high frequency, LP field being modeled by the periodically delta-kicked rotor. The kicked rotor is a theoretical model much used in quantum chaos studies, and it is known to be a close analog $[114,131,132]$ to the tight-binding model in solid state physics for a periodic Id lattice perturbed by disorder that causes Anderson (exponential) localization of the electronic wave function. Another experimental realization of quantal kicked-rotor dynamics uses laser cooled sodium atoms [133-135].

Other theoretical explanations for enhanced high-frequency stability of the quantal driven atom vs. its classical counterpart have been based on uncertainty principle violation [136]; dynamics in a severely truncated, quasiresonant state basis [19, 137-139]; and quantal vs. classical transport through cantori $[140,141]$, but see Secs. 7.4 and 9.4 of [127] for critical comments.

Regime-VI: the photoelectric effect. When the photon energy rises above the threshold for one-photon ionization, $\hbar \omega>\left(2 n_{0}^{2}\right)^{-1}$, all vestiges of classical behavior are gone, at least for weak field intensities. Theoretically, this is predicted not to be so for intensities high enough for so-called stabilization phenomena to come into play; see papers in [142] and references therein.

The first experimental evidence for adiabatic stabilization of Ne Rydberg atoms in the $5 g$ circular state in a $623 \mathrm{~nm}$ pulsed laser field was recently published $[143,144]$.

\subsection{Classical phase-space portraits}

Phase portraits of the driven classical motion of a $1 \mathrm{~d}$ hydrogen atom are a useful aid for interpreting the LP experimental results. Figure 3 shows one made for amplitude $F=3.14 \mathrm{~V} / \mathrm{cm}$ and $\omega / 2 \pi=9.923 \mathrm{GHz}$ with use of a computationally efficient, area-preserving, implicit, iterated mapping that is an excellent 


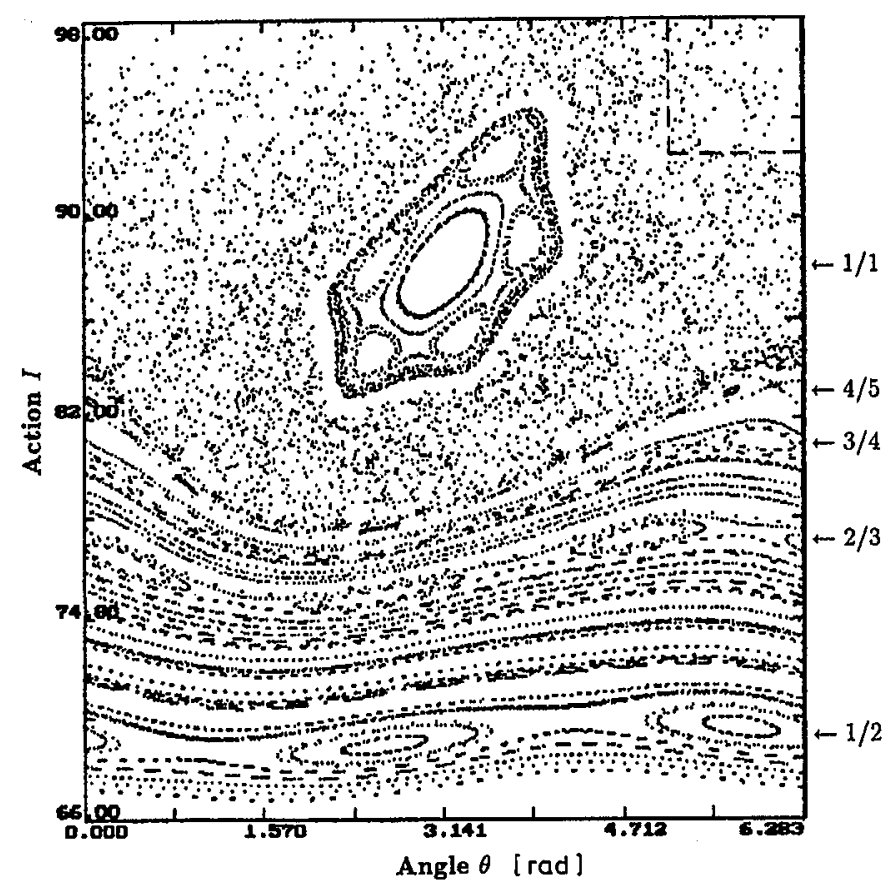

Fig. 3. Phase portrait (Poincaré surface-of-section) for the periodically driven 1d classical hydrogen atom obtained with use of an area-preserving, iterated mapping; see the text. It is an excellent approximation to the continuous dynamics for the scaled frequency $\Omega_{0}=I^{3} \omega$ above about 0.4 , here being used to record the evolution of 105 orbits having initial actions between 67.9 and 95.7 (in $\hbar$ ), for $F=3.14 \mathrm{~V} / \mathrm{cm}$ and $\omega / 2 \pi=9.903 \mathrm{GHz}$. The number of orbits and range of $\Omega_{0}$ sampled is enough to show several resonance zones for $\Omega_{0}=j / k$ from $1 / 2$ to $1 / 1$. Arrows along the right-hand vertical axis label them. Within each resonance zone, the local motion is pendulumlike and nearly regular, the more so far lower values of $I$. A region of irregular motion (sea of chaos) encircles the $1 / 1$ zone, which is also surrounded by a five-island-chain, secondary resonance zone that is born from an orbit bifurcation found numerically to occur near $2.96 \mathrm{~V} / \mathrm{cm}$. The dashed box in the upper right-hand corner shows size of Planck's quantum of action $h$.

approximation to the continuous dynamics ${ }^{\ddagger}$ for $\Omega_{0}$ above about 0.4 . Note that classical scaling of the dynamics [1] means that if classical dynamical evolution were calculated for different values of $I_{0}, \omega$, and $F$ that give the same ranges of $\Omega_{0}=I_{0}^{3} \omega$ and $F_{0}=I_{0}^{4} F$ used to make Fig. 3 , the classical phase portrait would be identical. However, the area of Planck's constant at fixed $\Omega_{0}$ and $F_{0}$. would change, becoming larger as ( $I_{0}$ decreases, $\omega$ increases, $F$ increases) and becoming smaller

tThe mapping used is equivalent to Eqs. (14)-(19) in Ref. [145a], except for adding a missing factor $\sin \left(\theta_{k}\right)$ that was inadvertently omitted from the right-hand side of Eq. (15) and for changing from positive to negative the signs in the right-hand-most terms of its Eqs. (14), i.e., the ones containing the symbol $\Phi_{0}$; this serves only to move the stable, elliptic fixed points of the primary resonance islands to $\theta=\pi$, in accord with the mapping Eqs. (15) in Ref. [145b]. 
as ( $I_{0}$ increases, $\omega$ decreases, $F$ decreases). As is reviewed in $[1,2]$, the effective Planck's constant is $\tilde{h}=h / n_{0}$; see also Secs. 1 and 5 .

Five different resonance zones are labeled by their respective rational values of the scaled frequency $I^{3}{ }_{\mathrm{r}} \omega=j / k$ associated with a resonant action $I_{\mathrm{r}}$. (In a $9.923 \mathrm{GHz}$ field, $I_{\mathrm{r}}=87.2$ gives $I_{\mathrm{r}}^{3} \omega=1$.) In a rotating frame the local motion within and near each zone is pendulumlike: librational motion around an elliptic fixed point (corresponding to a stable periodic orbit of the full motion) inside the zone and rotational motion outside the zone on either side of a separatrix (broken by the chaos-producing, higher-order terms beyond the pendulum approximation to the Hamiltonian) that connects to a hyperbolic fixed point (corresponding to an unstable periodic orbit of the full motion). The formula (see [87])

$$
I_{\mathrm{s}}^{ \pm}=\left(1 \pm 0.419 \sqrt{F_{\mathrm{r}}}+0.144 F_{\mathrm{r}}+\ldots\right) I_{\mathrm{r}},
$$

where $F_{\mathrm{r}}=I_{\mathrm{r}}^{4} F$, gives estimates for the maximal upward $I_{\mathrm{s}}^{+}$and downward $I_{\mathrm{s}}^{-}$excursions in action of the separatrix of the $1 / 1$ resonance zone. For the parameters used to make Fig. $3, I_{\mathrm{s}}^{+}=94.5$ and $I_{\mathrm{s}}^{-}=80.8$, so in the pendulum approximation the separatrix contains $\left(I_{\mathrm{s}}^{+}-I_{\mathrm{s}}^{-}\right)=13.7$ units of area measured in Planck's constant $h$. This gives a semiclassical estimate for the number of quantal states influenced by the main resonance zone at $\omega / 2 \pi=9.923 \mathrm{GHz}$ and $F=3.14 \mathrm{~V} / \mathrm{cm}$.

One may see by eye that far smaller area and, therefore, far fewer quantal states are associated with the $1 / 2,2 / 3,3 / 4$, and $4 / 5$ resonance zones. For formulae used to estimate their areas, see [146].

The full, driven classical motion is nonintegrable but only partly so when parameter values are chosen to give a phase portrait of coexisting regular and irregular (chaotic) regions, i.e., a mixed phase space. Each separatrix becomes, in fact, a chaotic layer whose thickness grows with increasing $F$ and within which are other fixed points with small resonance zones and chaotic layers, ad infinitum; see, e.g., p. 810 and Fig. 21 of [104]. Moreover, orbit bifurcations at certain values of the driving field amplitude produce new fixed points, resonance zones, and chaotic layers. Several such bifurcations occur for the main resonance zone at values of $F$ below that used to make Fig. 3. (See also [28] and [147].) Surrounding the $1 / 1$ resonance zone is a chaotic sea produced by higher-order terms beyond the pendulum approximation to the Hamiltonian. These terms mutate the separatrix into stable and unstable manifolds, converting its environs to a "sea of chaos". In this chaotic sea irregular orbits can diffuse to arbitrarily high action if the interaction time is sufficiently long. Indeed, the onset of global chaos is a classical mechanism for ionization.

One also sees in the phase portrait rotational Kolmogorov-Arnold-Moser (KAM) curves (tori) $[100,103,104,148,149]$ that are impenetrable barriers to transport in a periodically driven, one degree of freedom system; many such tori separate the $1 / 2$ and $2 / 3$ resonance zones.

Above it was remarked that the mapping used to make.Fig. 3 is an excellent approximation to the continuous, periodically forced dynamics in $1 \mathrm{~d}$ for $\Omega_{0}$ above about 0.4 . At lower $\Omega_{0}$ the approximations used to derive the mapping break down, but there is a deeper reason why not only it but phase portraits in general cease to be very useful at low $\Omega_{0}$. As $\Omega_{0}$ decreases, the time during which the electron 
can be accelerated in a given direction by the external LP field increases. This means that the momentum impulses given to the electron by the external field get larger, so large that the notion of ionization occurring classically by a diffusive wandering (net) upward in the phase space no longer makes sense. Moreover, as $\Omega_{0}$ decreases, the separation in action of the $1 / k$ (subharmonic for $k>1$ ) resonance zones decreases, but with increasing $F$, their widths explode as $F^{k / 2}[43,146,150]$.

At low $\Omega_{0}$ it makes more sense to think of the ionization as taking place by escape over a slowly oscillating barrier in coordinate space.

\subsection{LP results: old vs. new}

Figure 4 compares the scaled $10 \%$ - and $90 \%$-thresholds $F_{0}(0.1)$ and $F_{0}(0.9)$, respectively, for the $9.923 \mathrm{GHz} \mathrm{LP}$ data set from [1] with those from the present $9.904 \mathrm{GHz}$ LP data set $[81,82]$ obtained with the cavity described in Sec. 2.2. The

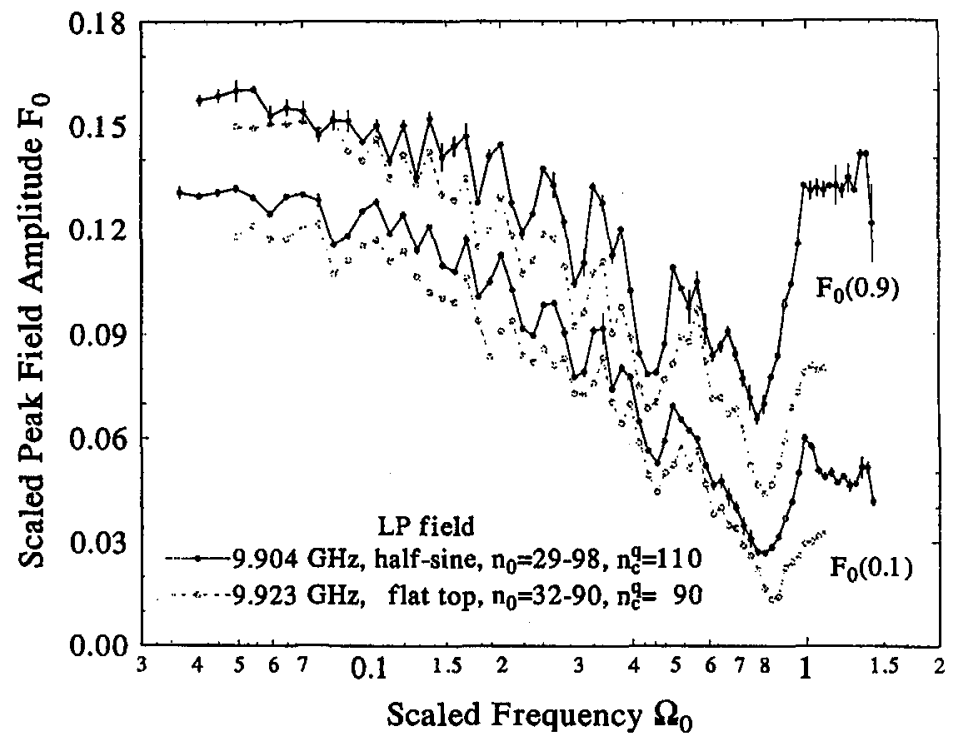

Fig. 4. A comparison of two different sets of LP microwave ionization data; see the text.

gross behavior of the two data sets is similar, but one notable, global difference is the latter data being systematically higher than the former data. The two most important contributions to this are undoubtedly

(i) the significantly shorter peak-field interaction time in the half-sine pulse envelope used to obtain the latter data, viz., above $95 \%$ peak value for only 31 field oscillations, compared to 230 oscillations for the flat-top envelope used to obtain the former data;

(ii) the significantly higher $n$ cutoff $n_{\mathrm{c}}^{\mathrm{q}} \approx 110$ used for the latter data, compared to $n_{c}^{q} \approx 90$ used for the former data. One expects that this effect would be greatest as $n_{0}$ for the former data set approaches $n_{\mathrm{c}}^{\mathrm{q}} \approx 90$; this is just what 
one sees in Fig. 4. (See the similar discussion in Sec. 3.5 of [1] about its Fig. 15.)

Because the 59 oscillation turn-on and turn-off ( $5 \%$ to $95 \%$ of maximum) used for the $9.904 \mathrm{GHz}$ data was comparable to the 80 oscillation turn-on and 95 oscillation turn-off used for the $9.923 \mathrm{GHz}$ data, i.e., both were "slow", it is less likely that they contributed to the differences in the data sets.

Notice the similar, local, undulatory structure in both data sets. As was explained above, the maxima are caused by the stabilizing influence of resonance zones centered at $\Omega_{0}=j / k$. In the $9.904 \mathrm{GHz} F_{0}(0.1)$ and $F_{0}(0.9)$ data, there are clear peaks near $\Omega_{0}=j / k$ for $k=1,2,3,4,5,6$ with $j=1$, as well as peaks near $2 / 5$ and $2 / 3$.

Using Eq. (14) of Ref. [1] to estimate the area in units of $h$ inside each $\Omega_{0}=$ $1 / k$ resonance zone, we obtain a semiclassical estimate of the number $N$ of quantal states inside. Taking experimental values $F_{0}(0.1)$ from the $9.904 \mathrm{GHz} \mathrm{LP}$ data, the formula gives $(N ; 1 / k)=(18.6 ; 1 / 1),(7.1 ; 1 / 2),(4.5 ; 1 / 3),(2.6 ; 1 / 4),(2.0 ; 1 / 5)$, and $(1.4 ; 1 / 6)$. This gives a neat quantal/classical correspondence: local maxima in experimental ionization thresholds occur close to $\Omega_{0}=1 / k$ when the classical resonance zones centered at these values contain at least one quantal state, i.e., when $N \gtrsim 1$.

Note that local maxima in $F_{0}(0.1)$ and $F_{0}(0.9)$ persist to lower values of $\Omega_{0}$, but these no longer line up with $\Omega_{0}=1 / k$ for $k \geq 7$. This could be explained, experimentally, by the discrete sampling of $\Omega_{0}$ with fixed $\omega$ and integer values of $n_{0}$ and, theoretically, by the classical resonances getting ever closer as $k$ increases and by $N$ dropping below one for $k \geq 7$. For $F_{0} \approx 0.12$, the formula gives, e.g., $(0.7 ; 1 / 7)$ and $(0.6 ; 1 / 8)$.

Why are the local maxima in the $9.904 \mathrm{GHz}$ data more pronounced than in the $9.923 \mathrm{GHz}$ data? Reason (i) above likely contributes because the shorter the interaction time, the larger the peak field amplitude can be before raising $P_{\text {ion }}$ to a given level. Because $N$ for classical $\Omega_{0}=1 / k$ resonances grows roughly as $F_{0}^{k / 2}$, see, e.g., Eq. (14) of [1], one expects their stabilizing influence to grow as $F_{0}$ grows, as long as the resultant increase in $P_{\text {ion }}$ is offset by the shorter interaction time. One expects reason (ii) above to contribute strongly as $n_{0}$ approaches $n_{\mathrm{c}}^{\mathrm{q}}$. This likely explains why the $\Omega_{0}=1$ resonance zone centered near $n_{0}=87$ is less prominent in the $9.923 \mathrm{GHz}$ data, for which $n_{\mathrm{c}}^{\mathrm{q}} \approx 90$ is only slightly higher. See also the discussion for regime-III in Sec. 3.1.

In both data sets, there are local maxima at other values of $\Omega_{0}$. In Sec. 5 we shall explicitly comment on the peak near $\Omega_{0}=1.3$. As may be seen by comparing experimental data and 3dCL in Fig. 2, the LP peak near $\Omega_{0}=1.3$ is not due to a classical 4/3 resonance zone. Rather, as was discussed for regime-IV in Sec. 3.1, this nonclassical peak is associated with a quantal separatrix state.

\section{Polarization dependence at low scaled frequencies}

As was noted in Sec. 2.3, the CP ionization curves in Fig. 1 stretch out compared to those for other polarizations. We now explain why this happens when $\Omega_{0}$ is low $[29,83]$. First we need to recall well-known features of ionization of excited hydrogen atoms by a static, an LP, and a CP field. Hereafter using 
a.u. unless explicitly noted, the Hamiltonian in the laboratory coordinate frame $(X, Y, Z)$ is $\mathcal{H}(t)=p^{2} / 2-1 / R+\boldsymbol{R} \cdot \boldsymbol{F}(t)$. For a static field, $\boldsymbol{R} \cdot \boldsymbol{F}(t)=Z F$, the system is separable in parabolic coordinates $(\xi, \eta, \phi)[151]$. Below the onset of ionization, all three classical actions $I \equiv\left(I_{\xi}, I_{\eta}, I_{m}\right)$ [quantum numbers $\left(n_{1}, n_{2}, m\right)$ ] are conserved. Classically, there is a sharp threshold field $F_{\text {crit }}(I)$ below which the motion with these actions remains bound; from the least robust orbit [quantally, $m=n_{1}=0, n_{2}=(n-1)$ ] to the most robust orbit $\left[n_{1}=(n-1), n_{2}=m=0\right], n_{0}^{4} F_{\text {crit }}$ varies between about $0.13-0.38$. Tunneling through the $\eta$-barrier allows ionization for $F<F_{\text {crit }}$; for interaction times near $10^{-8} \mathrm{~s}$ and $n_{0} \approx 40$, it lowers thresholds by $10-15 \%[1,152]$.

For an LP field, $\boldsymbol{R} \cdot \boldsymbol{F}(t)=\lambda(t) Z F \sin (\omega t+\psi)$, with $\psi$ an initial phase; again, separability leads to conservation of $m$. The dynamics is quasistatic [1, 94] if $\Omega_{0}$ is sufficiently small and away from exponentially sharp resonances discussed theoretically for $1 \mathrm{~d}$ in [43]. The spatial reversal of $F$ on each half-cycle interchanges $n_{1}$ and $n_{2}$; therefore, as Fig. 1 of [94] shows, for a uniform mixture of substates of a given $n_{0}$, classically a microcanonical ensemble, the ionization probability $P_{\text {ion }}$ rises from 0 to 1 as the scaled amplitude $F_{0}$ varies between about $0.115-0.17$. This is just the beginning of the wider classical range, $F_{0}=0.13-0.38$, lowered $10-15 \%$ by tunneling, discussed above for the static field case.

In a frame $(x, y, z)$ rotating at frequency $\omega$, the Hamiltonian is given by

$$
\begin{aligned}
& K(t)=p^{2} / 2-1 / r+\lambda(t) \mathcal{F} y+\omega L_{z} \\
& +0.5 \lambda(t) \mathcal{F}[y \sin 2 \omega t-x(1-\cos 2 \omega t)] \sin \delta_{1} \\
& +0.5 \lambda(t) \mathcal{F}[x \sin 2 \omega t-y(1+\cos 2 \omega t)] \sin ^{2}\left(\delta_{1} / 2\right),
\end{aligned}
$$

where $\delta_{1}=\pi / 2-\delta$ measures the deviation from CP.

For CP the Hamiltonian in the rotating frame consists of the first line in Eq. (3), which represents the free hydrogen atom perturbed by a static field term and the Coriolis term $\omega L_{z}$. (This CP Hamiltonian is close to one giving integrable motion [70-72].) For $\omega$ low enough one expects that the Coriolis term should have little effect on the ionization dynamics. References $[29,83]$ confirmed that it affected $P_{\text {ion }}$ very little for $\Omega_{0} \lesssim 0.1$ by finding close similarity between classical trajectory ionization curves calculated with (the CP case) and without (the static field case) the $\omega L_{z}$ term. Therefore, at low $\Omega_{0}$ the lack of interchange of $n_{1}$ and $n_{2}$ (see above) should cause quantal CP ionization curves to stretch out and approach $P_{\text {ion }}=1$ at a higher value of $F_{0}$ than for the LP case.

Though this explains the behavior of the CP curves in Fig. 1, this is not the only polarization-dependent difference. Note the different shape of the $n_{0}=42 \mathrm{EP}$ curve for $\delta=0.45 \pi$. Figure 5 shows the EP dependence for $n_{0}=42$ over a much finer range, from $\delta=0.42 \pi$ to $0.5 \pi$, with an experimental uncertainty $\Delta \delta=0.01 \pi$. Here there is a remarkable local variation of $P_{\text {ion }}$ with $\delta$ at fixed $F_{0}$. Over about the same range of $\delta$, similar EP dependence was observed for $n_{0}=41$ and 43 (data not shown here), but not for $n_{0} \gtrsim 44$ nor, with exceptions discussed below, for $n_{0} \lesssim 40$.

We now extend the discussion above for the CP case to give a classical explanation for the physical origin of the sensitivity of $n_{0}=41,42,43$ at $9.904 \mathrm{GHz}$ to 


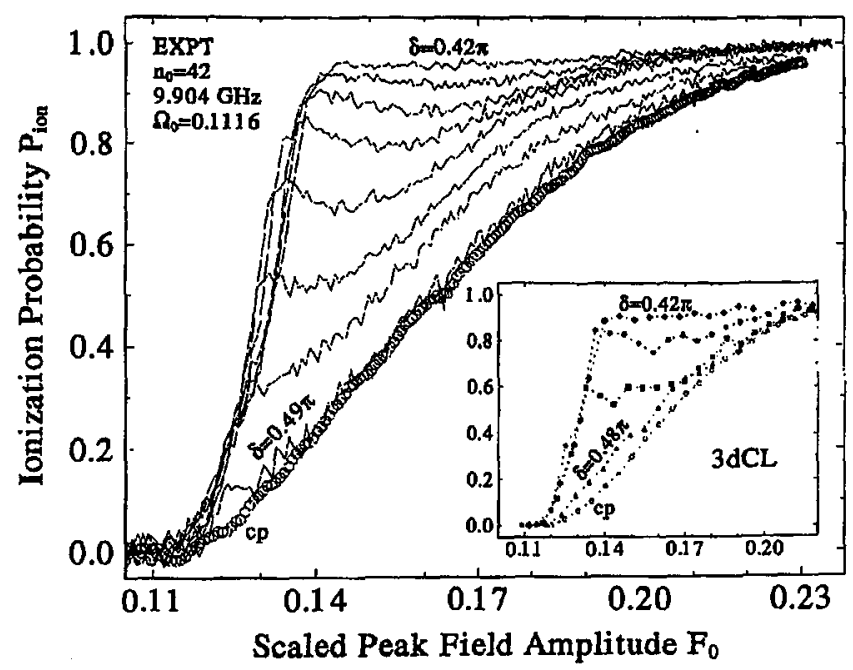

Fig. 5. The detailed elliptical-polarization dependence for $n_{0}=42$ in steps of $\delta / \pi=0.01[\delta / \pi=0.02]$ for experimental data [for 3dCL calculations (inset)].

EP for values of $\delta$ close to CP; mathematical details of the theory are given in [29]. A full understanding of the polarization-dependent dynamics will require $3 \mathrm{~d}$ quantal calculations, but state-of-the-art 3d quantal (Floquet) calculations have been reported, so far, only for LP and CP and for $n_{0} \lesssim 23$ [153]. Until improvements in computers and algorithms enable $3 \mathrm{~d}$ quantal calculations for $3 \mathrm{~d}$ hydrogen atoms in an EP field, with the atomic and field parameters used for the present experiments, we must use insights gleaned from comparisons of experimental data with classical calculations and theory.

We begin with 3d classical Monte Carlo calculations (3dCL), the results of which $[29,83]$ are shown in the inset to Fig. 5. Allowing for the $\pm 0.01 \pi$ experimental uncertainty in $\delta$, there is remarkable, quantitative agreement between the experimental data and 3dCL; similar agreement was obtained for $n_{0}=41$ and 43 , not shown here.

This agreement validates the use of classical theory to understand the extreme local sensitivity of the ionization dynamics to polarization. But we need to understand what produces the local maximum in $P_{\text {ion }}$ at, e.g., $F_{0} \approx 0.13$ for $\delta \approx 0.45 \pi$ and why it has this effect. Because it involves the complexities of multidimensional dynamics, the discussion here can only be brief; for the details see [29]. The key point is that all perturbations vary little during an unperturbed Kepler period, so one can average over this fast motion, reducing the number of degrees of freedom, and then write the resulting equations for the mean motion in terms of the vectors $\boldsymbol{X}=\boldsymbol{L}-\boldsymbol{A}$ and $\boldsymbol{Y}=\boldsymbol{L}+\boldsymbol{A}$ where $\boldsymbol{L}$ is the orbital angular momentum and $\boldsymbol{A}=(p \times \boldsymbol{L}-r / r) / \sqrt{-2 E}$ is the Runge-Lenz vector. For a CP field $\left(\delta_{1}=0\right)$, both $\boldsymbol{X}$ and $\boldsymbol{Y}$ rotate uniformly about the field direction with the scaled frequency $\bar{\omega}=\sqrt{\omega_{\mathrm{S}}^{2}+\Omega_{0}^{2}}$, where $\omega_{\mathrm{S}}=3 \mathcal{F}_{0} / 2$ is a classical Stark frequency associated with the scaled amplitude $\mathcal{F}_{0}$. For the ranges of $F_{0}$ and $\Omega_{0}$ considered here, $\bar{\omega}<\frac{1}{3}$. 
Perturbations of frequency $2 \Omega_{0}$ and strength proportional to $\delta_{1}$, which appear for an EP field, can resonate with the mean motion when $\bar{\omega}=2 \Omega_{0}$, i.e., when $\mathcal{F}_{0} \approx \mathcal{F}_{0_{\mathrm{r}}} \equiv 2 \Omega_{0} / \sqrt{3}$. For $\Omega_{0}=0.1116$ this gives $\mathcal{F}_{0_{\mathrm{r}}}=0.13$; equivalently, this is $F_{0_{\mathrm{r}}}$ varying from 0.145 to 0.13 as $\delta / \pi$ varies from 0.42 to 0.5 , in agreement with the local maxima in Fig. 5 . We use " $2 \Omega_{0}$ resonance" to label this dynamics.

The averaged equations of motion give a model for understanding how the $2 \Omega_{0}$ resonance affects the bound-state dynamics. With the full richness of the details being given in [29], ionization is added post hoc to the model via the time dependence of $\mathcal{F}_{\text {crit }}(t)=\mathcal{F}_{\text {crit }}(\boldsymbol{I}(t))$, which can be used to mimic the classical escape over the barrier when $\mathcal{F}_{\text {crit }}(t)<\lambda(t) \mathcal{F}$. (Recall that $0 \leq \lambda(t) \leq 1$ is the pulse envelope.) This model, compared with exact calculations in [29], shifts one's attention to understanding the temporal evolution of the classical actions $\boldsymbol{I}(t)$ and critical fields $\mathcal{F}_{\text {crit }}(\boldsymbol{I}(t))$. Though the details are complicated, the analysis shows that if $I_{m}>0[<0]$ then $\mathcal{F}_{\text {crit }}(t)$ decreases [increases] as $\lambda(t) \mathcal{F}$ increases through the resonance by an amount which increases as $\mathrm{d} \lambda / \mathrm{d} t$ decreases. Numerical calculations show that the mean over $I_{m}>0$ and $I_{m}<0$ is dominated by the behavior of the former, thereby producing the observed local maximum in the ionization probability $[29,83]$.

Data for $n_{0}=31,32,33$ also exhibited extreme sensitivity to EP, but for lower values of $\delta$ than for the $2 \Omega_{0}$ resonance. Figure 6 shows results for $n_{0}=31$ for eight values of $\delta$ between $0.28 \pi-0.45 \pi(F / \mathcal{F}$ between 1.28-1.08). Observe the clear similarity between Figs. 6 and 5 . The inset shows that the 3dCL results, obtained for the same eight values of $\delta$, again reproduce the experimental polarization dependence.

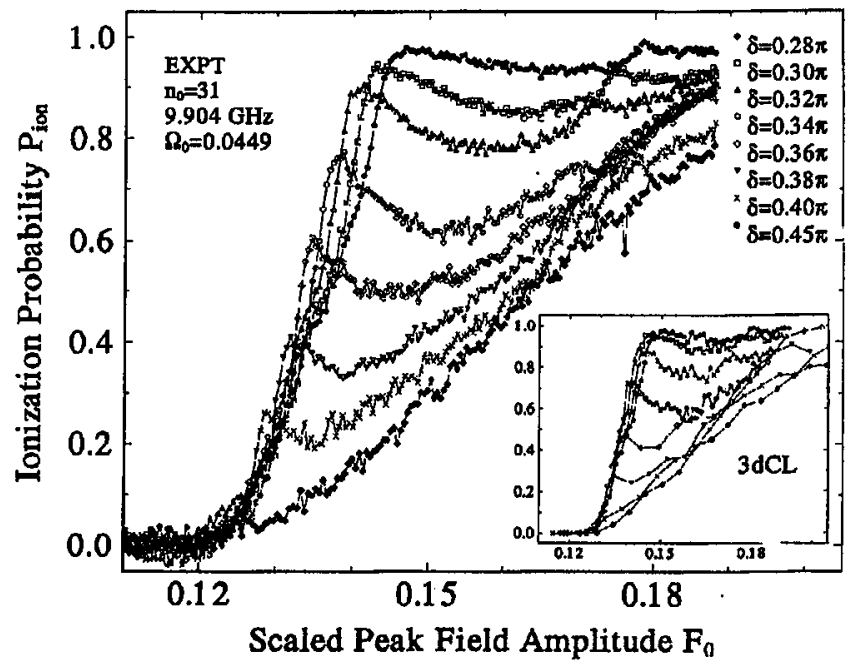

Fig. 6. The detailed elliptical-polarization dependence for $n_{0}=31$ for experimental data [for 3dCL calculations (inset)]. 
Though $K(t)$, Eq. (3), has harmonic terms only at $2 \Omega_{0}$, let us, nevertheless, extrapolate the frequency-matching condition to a " $4 \Omega_{0}$ resonance": $4 \Omega_{0}=\bar{\omega}=$ $\sqrt{\left(3 \mathcal{F}_{0} / 2\right)^{2}+\Omega_{0}^{2}}$, giving $\Omega_{0}=0.39 \mathcal{F}_{0}$. The classical static field ionization threshold, $\mathcal{F}_{0}=0.13$, gives $\Omega_{0}=0.05$; at $9.904 \mathrm{GHz}$ this gives $n_{0}=32$, the middle of the three $n_{0}$-values where extreme sensitivity to EP was observed $[81,83]$. This result cannot be accidental. It predicts that successive application in the rotating frame of perturbation theory, classical or quantal, will lead to resonances at even powers of $\Omega_{0}$. The detailed derivation has been accomplished, so far, only classically and only for the $2 \Omega_{0}$ resonance [29].

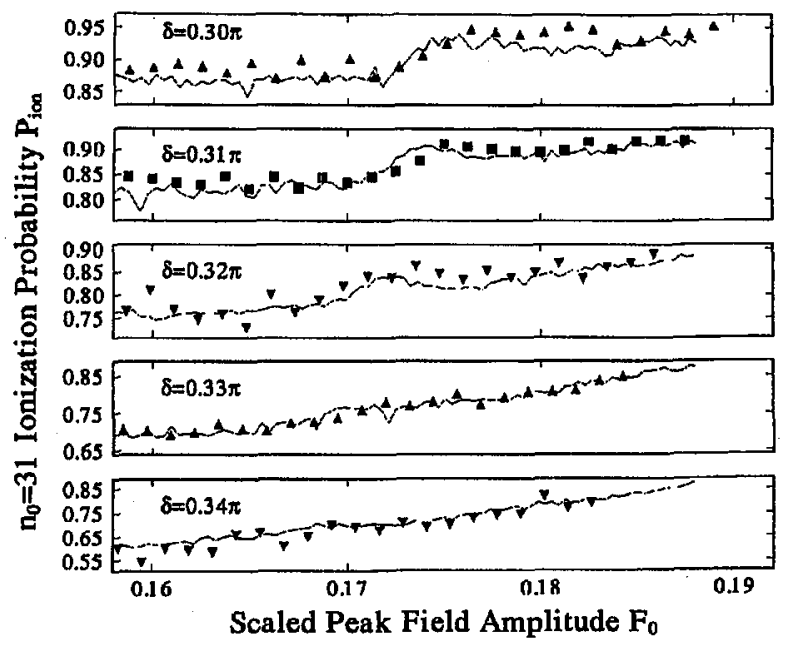

Fig. 7. The detailed elliptical-polarization dependence for $n_{0}=31$ for experimental data (solid curves) and for 3dCL calculations (inverted filled triangle: 422 orbits; upright filled triangle: 864 orbits; filled square: 1728 orbits).

The top three curves in Fig. 6 show another rise in $P_{\text {ion }}$ near $F_{0}=0.17$ and a subsequent plateau. For five values of $\delta / \pi$ in steps of 0.01 , Fig. 7 compares experimental data in this region with the results of $3 \mathrm{dCL}$. The rise and subsequent plateau in the data for $\delta / \pi=0.30,0.31,0.32$, as well as its disappearance for higher values of $\delta / \pi$, is quantitatively reproduced by 3dCL. Arguments were given in [83] that this behavior is caused by the " $6 \Omega_{0}$ resonance".

Therefore, these low frequency experiments, supported by $3 \mathrm{dCL}$, establish a series of EP-induced resonances that occur when even integer powers of the scaled driving frequency $\Omega_{0}$ match the frequency of the mean classical Stark motion in the rotating frame. Such resonances are a striking example of the kind of the richness that can occur in the higher-dimensional dynamics $(3 \mathrm{~d}+$ time) of a driven quantal system. In this case, even classical theory quantitatively reproduces the behavior and provides a clear physical picture for what causes it. As was emphasized in [83], this EP-induced resonant dynamics provides a mechanism for controlling the ionization. 


\section{Polarization dependence near the main resonance zone}

In the preceding section we saw that the polarization dependence of the microwave ionization of excited hydrogen atoms can be dramatic at low scaled frequencies. We now examine the polarization dependence for hydrogen atoms with $n_{0}=70, \ldots, 98$ at $9.904 \mathrm{GHz}$, which corresponds the range of scaled frequency $\Omega_{0}=0.517-1.417$. This includes the main resonance zone centered at $\Omega_{0}=1$, which, we shall see, exerts a controlling influence that makes the ionization dynamics near the onset of ionization independent of polarization. when a certain classically derived, amplitude-scaling of the field is used.

The experimental techniques described in Sec. 2 were used to measure $[81,82]$ $9.904 \mathrm{GHz}$ ionization curves for LP, CP, and a number of different values of EP. From these curves were obtained the $10 \%$ and $50 \%$ ionization thresholds plotted in Fig. 8 with use of scaled parameters. (Please note the suppressed zeros on the vertical axes of panels (a) and (b).) Focusing on Fig. 8a, note that the dependence of $F_{0}(0.1)$ on $\Omega_{0}$ has the same shape for all three polarizations. Also note that the onset of ionization for CP in this range of $\Omega_{0}$ occurs at lower peak field amplitude $F_{0}$ than it does for LP. This behavior is opposite to the rule of thumb mentioned in Sec. 1 that emerged from laser multiphoton ionization experiments with tightly-bound atoms.
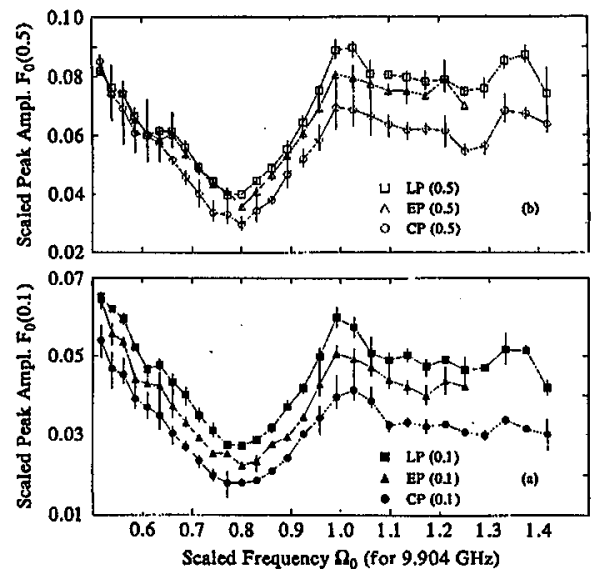

Fig. 8. Frequency- and polarization-dependence of experimental ionization peak-field thresholds (filled symbols, $P_{\text {ion }}=0.1$; open symbols, $\left.P_{\text {ion }}=0.5\right)$ for $\mathrm{H}\left(n_{0}=70, \ldots, 98\right.$ ) atoms.

As was explained in Sec. 3 , because the local maximum near $\Omega_{0}=1$ is known for the LP case to be due to the stabilizing effect of the main resonance zone, the experimental results show this effect is independent of polarization.

This is an important result for the following reason. Using lower case $d$ (upper case D) to represent spatial (phase-space) dimension, the phase portrait in Fig. 3 is a result of following $1 \mathrm{~d}$ dynamics that is periodically forced in time, visualized stroboscopically in a $2 \mathrm{D}$ plot. Colloquially, such dynamics is said to 
have $1 \frac{1}{2}$ degrees of freedom. For a $3 \mathrm{~d}$ atom in the time-dependent driving field, the dimensionality depends on the polarization. For LP, the conservation of the projection of the orbital angular momentum on the field polarization axis reduces the dimensionality to $2 \mathrm{~d}+$ time, or $5 \mathrm{D}$, or $2 \frac{1}{2}$ degrees of freedom. Because of a separation of time scales [117], the LP dynamics is well approximated near the onset of ionization by the $(1 \mathrm{~d}+$ time $)$ model $\left(1 \frac{1}{2}\right.$ degrees of freedom). This explains why the surface-state-electron (SSE) model, reviewed in [19], has been able to provide good estimates for onsets of ionization measured with $3 \mathrm{~d}$ hydrogen atoms in an LP field, at least for $\Omega_{0}$ up to 2.8 or so [154]. But note that it has been predicted $[24,27]$ that the SSE model may not provide quantitatively useful 1d estimates for the LP ionization thresholds for $3 \mathrm{~d}$ hydrogen atoms at much higher scaled frequencies.

The EP dynamics is $3 \mathrm{~d}+$ time, or $7 \mathrm{D}$, or $3 \frac{1}{2}$ degrees of freedom.

Let us look back to Eq. (3), the Hamiltonian $K(t)$ in the frame $(x, y, z)$ rotating at frequency $\omega$. For $\mathrm{CP}, \delta_{1}=0$, and ignoring the slow pulse envelope $\lambda(t)$, the time dependence is removed, but there remains the static Stark term $\mathcal{F} y$ and the Coriolis term $\omega L_{z}=\omega\left(x p_{y}-y p_{x}\right)$. In contrast to the discussion in Sec. 4, because we are now considering scaled frequencies that are not low, we cannot ignore the Coriolis term. That it mixes coordinates and momenta means that the concept of potential energy surface no longer applies, though one can use so-called zero-velocity curves as an effective potential $[53,63]$.

Figure 9 compares the experimental data shown in Fig. 8 with the results of $3 \mathrm{dCL}$ [82] that modeled all important features of the experiment, including the uniform distribution of initial substates, the pulse envelope $\lambda(t)$, and the $n$ cutoff $n_{c}^{\mathrm{q}}$. (See also Fig. 2.) The accord between experiment and 3dCL is equally good for CP, EP, and LP, with wide regions of striking agreement and those of disagreement being similarly localized for all polarizations. Systematically, the 3dCL lie below experimental data (a) for $\Omega_{0} \gtrsim 1.15$ and (b) for $0.8 \lesssim \Omega_{0} \lesssim 0.9$. Using in Eq. (2) the coordinates for the local maximum in the LP data at $\left(\Omega_{0}, F_{0}(0.1)\right) \approx(1.33,0.052)$ gives $I_{s}^{+}=95$ for the location of the upper separatrix of the pendulum, in good agreement with the local maximum in the LP data at $n_{0}=96,97$. Similarly, on the lower side of the $\Omega_{0}=1$ resonance bump, using the coordinates in the LP data at $\left(\Omega_{0}, F_{0}(0.1)\right) \approx(0.083,0.028)$ gives $I_{\mathrm{s}}^{-}=81$ for the location of the lower separatrix of the pendulum; this is in good agreement with the largest local excursion at $n_{0}=82,83$ of the LP data above the $3 \mathrm{dCL}$ results, which is matched by excursions in the EP and CP data, too.

We interpret these results as demonstrating the polarization independence of the nonclassical, stabilizing influence of quantal separatrix states prepared in the experiment from both above and below the pendulumlike resonance zone, i.e., associated with both the upper and lower branches of the separatrix.

Note that the nonclassical stability near the upper branch of the separatrix occurs at $\left(n_{0} ; \omega / 2 \pi\right)=(96,97 ; 9.904 \mathrm{GHz})$. This may be compared to previous LP data $[33,87]$, where it occurred for $(62 ; 36.0 \mathrm{GHz}),(66 ; 30.36 \mathrm{GHz})$, and $(69 ; 26.4 \mathrm{GHz})$. That enhanced, nonclassical stability associated with quantal separatrix states persists from $36.0 \mathrm{GHz}$ to $9.904 \mathrm{GHz}$ shows that this semiclassical 


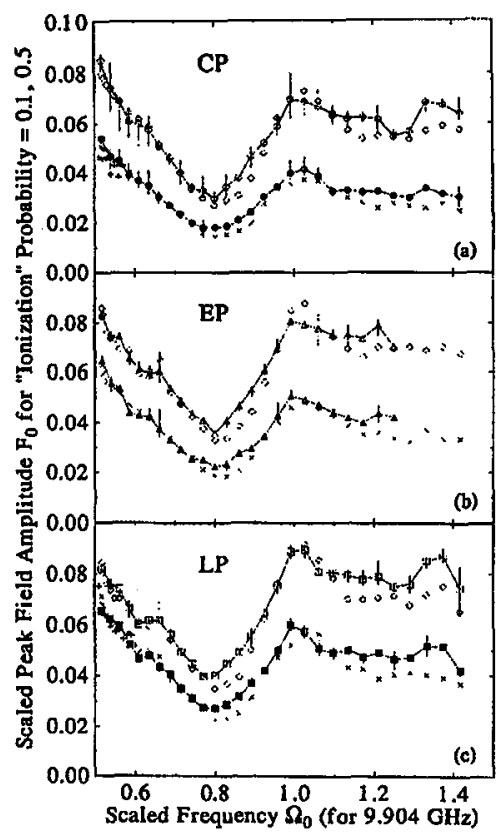

Fig. 9. The same data shown in Fig. 8 compared with the results of 3 d classical Monte Carlo simulations (X) for (a): CP; (b): EP; (c): LP; and with the results of $2 \mathrm{~d}$ classical Monte Carlo calculations (cross) for (a): CP and (c): LP. See the text.

effect [33] is insensitive to at least a $50 \%$ variation in the effective Planck's constant $\widetilde{\hbar}$; see Secs. 1 and 3.2 .

Other disagreements between experiment and 3dCL in Fig. 9 are localized near the top of the $\Omega_{0}=1$ resonance bump, but they are not systematic.

Also shown in Fig. 9 are the results for CP, frame (a), and LP, frame (c), of classical calculations [39] used to predict 10\%-ionization thresholds for LP and CP fields over the scaled frequency range $0.01 \leq \Omega_{0} \leq 0.6$; these were carried out before the present experiments on the polarization dependence of the microwave ionization of $3 \mathrm{~d}$ hydrogen atoms were done [81-83]. Let us refer to these calculations as $2 \mathrm{dCL}$ because they used only ensembles of orbits whose plane of motion included the microwave electric field vector. In these $2 \mathrm{dCL}$, unlike the subsequent experiments, the initial pricicipal action was held fixed, and the frequency $\omega$ was changed to vary $\Omega_{0}$. The pulse envelope used in the $2 \mathrm{~d} \overline{\mathrm{CL}}$ rose over 200 initial Kepler periods $T_{\mathrm{K}}$, remained flat for $40 T_{\mathrm{K}}$, and fell over $200 T_{\mathrm{K}}$. This is not too different from the experimental, half-sine pulse shape, see Sec. 2.2, whose 152 field oscillation duration corresponds to $294 T_{\mathrm{K}}$ at $n_{0}=70$, dropping to $107 T_{\mathrm{K}}$ at $n_{0}=98$. The reasonable agreement among the $2 \mathrm{dCL}$, the experimental data, and the 3dCL in Fig. 9, where they overlap, bears out our expectation that modest changes in the pulse shape will exert only a small influence on the ionization thresholds when $\Omega_{0}$ is not "too high". For additional $2 \mathrm{dCL}$ and a discussion of classical time scales for hydrogen atoms driven by a strong CP field at values of $\Omega_{0}$ below the main resonance zone, see [39]. 


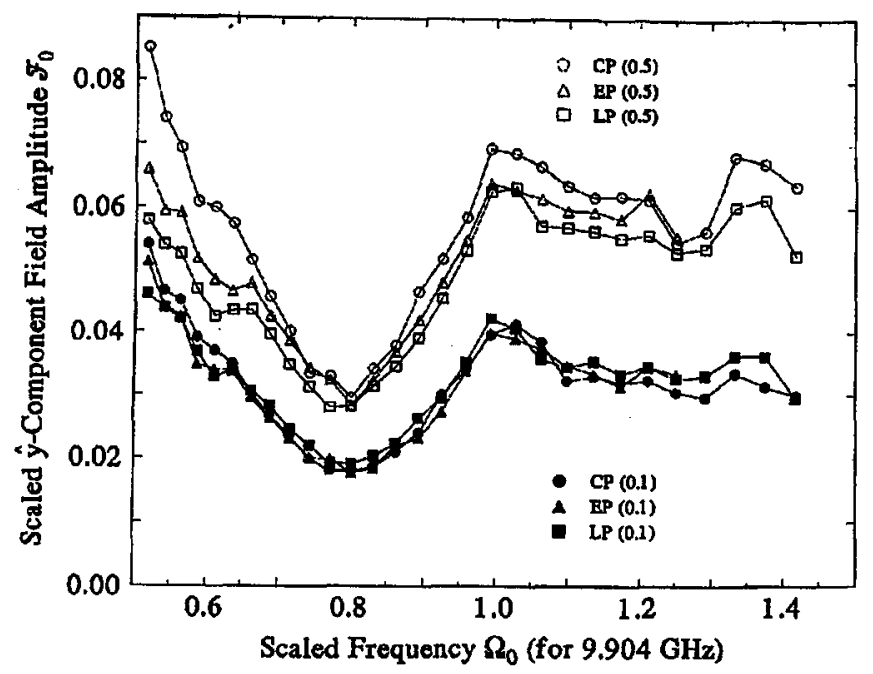

Fig. 10. Frequency- and polarization-dependence of the measured ionization thresholds expressed in terms of the scaled $\widehat{\boldsymbol{Y}}$-component field amplitude $\mathcal{F}_{0}=n_{0}^{4} \mathcal{F}$ defined in the text.

Figure 10 replots the experimental data in Figs. 8 and 9 using a field parameterized with the scaled $\widehat{\boldsymbol{Y}}$-component amplitude $\mathcal{F}$ for $\alpha=1$ and variable $\delta$; see Eq. (1) in Sec. 2.2. For our [LP] $\{E P\}$ (CP) data, this $\mathcal{F}$ is $[1 / \sqrt{2}]\{1 / 1.26\}(1.00)$ times the peak amplitude $F$. Plotted this way the classically scaled thresholds $\mathcal{F}_{0} \equiv n_{0}^{4} \mathcal{F}(X)$ vs. $\Omega_{0}$ are insensitive to polarization near the main resonance for low $X$; e.g., for $\Omega_{0}>0.63$ the $\mathcal{F}_{0}(0.1)$ thresholds are nearly identical for LP, EP and $\mathrm{CP}$. However, this agreement persists only up to $X=0.3$; at $X \gtrsim 0.5$ the $C P$ thresholds are systematically higher.

Encouraged by the agreement of the data with classical simulations, and realizing that the high values of $n_{0}$ and inherent $3 \mathrm{~d}$ nature of this problem strain the most advanced quantal computations [11, 76, 153], a classical theory was used [82] to understand these results; Ref. [155] gives details. Here we provide only a very brief qualitative explanation of why classical dynamics leads to the invariance of $\mathcal{F}_{0}(X)$ with respect to polarization, observed for $X \leq 0.3$, and how the main resonance causes it. The dynamics of the $3 \mathrm{~d}$ hydrogen atom is determined by the Hamiltonian $H=p^{2} / 2-1 / r+\boldsymbol{r} \cdot \boldsymbol{F}(t)$, where $\boldsymbol{F}(t)$ was given in Eq. (1). Using the unperturbed, $\boldsymbol{F}=0$, angle-action variables $\left(\theta_{n}, \theta_{l}, \theta_{m}, I_{n}, I_{l}, I_{m}\right)$ [156], one can show that near the principal resonance, where $\omega=\omega_{\mathrm{K}}\left(I_{n}\right)$, because $\dot{\theta}_{n} \approx \omega_{\mathrm{K}}$ the system has three distinctly different dynamical time scales: fastest is the field variation, slowest is the changing orientation of the Kepler ellipse, and intermediate is the change in $\theta_{n}$. It follows, see [155], that for $\omega \approx \omega_{\mathrm{K}}$ there is a canonical representation in which the Hamiltonian has the form $K=K_{\mathrm{p}}+K_{\mathrm{f}}$, where $K_{\mathrm{f}}$ is a rapidly varying part that causes the escape from the regular, slow motion due to $K_{\mathrm{p}}$.

The slow Hamiltonian, $K_{\mathrm{p}}$, may be approximated by one similar to that of a plane vertical pendulum subject to gravity, with a "gravitational" coefficient that 
is a function of the slowly varying angle-action variables

$$
K_{\mathrm{p}}=-\frac{3 p^{2}}{2 I_{\mathrm{R}}^{2}}+\lambda(t) \mathcal{F}_{0} H_{\mathrm{r}}(\boldsymbol{\Theta} ; \delta) \cos q
$$

where $\Theta=\left(\theta_{l}, \theta_{m}, I_{l}, I_{m}\right), I_{\mathrm{R}}$ is the value of the principle action satisfying $\omega=\omega_{\mathrm{K}}\left(I_{\mathrm{R}}\right)$, and $(q, p)$ are simply related to the variables describing the motion around the Kepler ellipse. Reference [45] analyzes motion produced by a Hamiltonian similar to $K_{\mathrm{p}}$, and Ref. [156] shows that $H_{\mathrm{r}}$ is an approximate adiabatic invariant and that the angles $\Theta$ vary more slowly than $(q, p)$ which, in turn, vary more slowly than $K_{\mathrm{f}}$. Moreover, $H_{\mathrm{r}}$ is relatively weakly dependent upon $\delta$, the field ellipticity; the mean of $H_{\mathrm{r}}^{2}$ over a microcanonical distribution is independent of $\delta$.

The slowly varying field envelope adds the final, and slowest, time scale that is important to the ionization dynamics [157]. Including its effect one can, e.g., estimate the ionization threshold at the minimum of $\mathcal{F}_{0}(X)$ near $\Omega_{0}=0.8$. For $\Omega_{0}<1$ the first orbits to ionize as $\mathcal{F}_{0}$ increases satisfy two conditions [22]: (i) they adiabatically switch onto the separatrix of $K_{\mathrm{p}}$ as $\lambda(t)$ slowly increases from 0 to 1 . (ii) The amplitude $\mathcal{F}_{0}$ must be large enough for the first $\left(\Omega_{0}=1\right)$ and second $\left(\Omega_{0}=2\right)$ resonance island to touch: this is the Chirikov overlap criterion [100]. (For recent classical theoretical work applying the resonance overlap criterion to the CP and EP cases, see [74] and [158], respectively.) When (i) is satisfied, the initial phase points gradually move onto the separatrix of $K_{\mathrm{p}}$ and then wind around the edge of the resonance island. The presence of $K_{\mathrm{f}}$ converts separatrices into stable and unstable manifolds. When (ii) is satisfied, a proportion of orbits transfers to the unstable manifold of the $\Omega_{0}=2$ island and subsequently to higher actions that easily ionize.

These two conditions provide estimates for the frequency of the minimum threshold, near $\Omega_{0}=0.8$, and also the critical values of $\mathcal{F}_{0}$ at which ionization first occurs, $\mathcal{F}_{0}^{\mathrm{c}}(\boldsymbol{\Theta}, \delta)$; Ref. [155] gives details. Since the angles $\boldsymbol{\Theta}$ are slowly varying functions of time, the minimum of this function gives an estimate of the critical field. This minimum turns out to be only weakly dependent upon $\delta$. As $\delta$ increases from 0 to $\pi / 2, \min \left(\mathcal{F}_{0}^{\mathrm{c}}(\boldsymbol{\Theta}, \delta)\right)$ decreases monotonically from about 0.014 to 0.011 and $\Omega_{0}^{\mathrm{c}}$ from 0.78 to 0.77 . In the $3 \mathrm{dCL}$ simulations the minimum of $\mathcal{F}_{0}(0.1)$ decreases from 0.016 to 0.014 , a statistically insignificant change, and $\Omega_{0}^{\complement} \approx 0.8$, for all $\delta$.

The analysis sketched above uses the approximate Hamiltonian $K_{\mathrm{p}}$ to give a theoretical explanation for the insensitivity to polarization of the ionization thresholds $\mathcal{F}_{0}(X=0.1)$ plotted in Fig. 10. Therefore, it seems clear that the pendulumlike resonance dominates the strong-field dynamics in the neighborhood of $\Omega_{0}$. Because such resonances are generic features of mixed-phase-space classical systems and have a clear quantal analog $[22,99,105,159]$, this result should not be regarded as a special case.

It is interesting to ask what determines the boundaries of the influence of the main resonance zone. We tentatively conclude that the marked divergence of the thresholds for different polarizations in Fig. 10 at $\Omega_{0} \approx 0.63$ is the signature of its lower boundary, but analysis to demonstrate this theoretically must be left for future work. It is tantalizing that this divergence might supply a sort of crude 
spectroscopy for experimentally determining where the boundaries of pendulumlike resonance zones occur in a quantal dynamical system. Because one expects the effect of quantal tunneling - now in phase space [160], not configuration space to blur whatever sharpness there is to classical barriers to transport, even a crude spectroscopic tool homes in on some very interesting physics.

\section{Conclusions}

The results of our first experimental forays [81-83] into the terra incognita of the polarization dependence of the microwave ionization of excited hydrogen atoms has presented some surprising similarities with the extensive body of previous LP results as well as a new discovery. The surprising similarities occur in the data presented in Sec. 5 for $0.6 \lesssim \Omega_{0} \lesssim 1.4$, a range that overlaps the near-classical regime-III and the transition regime-IV. When an amplitude scaling relation given by classical theory is used, the experimental data here show that, near onset, i.e., for $P_{\text {ion }} \lesssim 0.3$, the scaled ionization thresholds are approximately independent of polarization. The similarity of the EP and CP behavior with the LP behavior was an unanticipated surprise. Classical theory shows that it is a result of separation of time scales in a regime where the dynamics is influenced by the main resonance zone. For field amplitudes $\mathcal{F}$ raising $P_{\text {ion }}$ significantly above 0.3 , however, the simple similarity expressed by the theoretical amplitude scaling relation goes away. Perhaps the nature of the dynamics or its effective dimensionality change as $\mathcal{F}$ increases. We need to understand how the most easily ionized orbits (classically) or wave packets (quantally) differ from those that do not ionize until much higher values of $\mathcal{F}$. However, though the theoretical amplitude scaling relation breaks down for higher values of $\mathcal{F}$ and $P_{\text {ion }}$, the stabilizing influence of quantal separatrix state(s) above and below the main resonance zone does not. It will be fascinating to study and understand the detailed reasons for this behavior.

The new discovery is found in the data presented in Sec. 4 for $\Omega_{0} \lesssim 0.1$. For LP driving this range overlaps the low-frequency regime-II, which is defined by resonances between quantal adiabatic states of the (atom + field) system, and the quasistatic tunneling regime-I. For certain narrow ranges of $n_{0}$ at fixed driving frequency $\omega$, we find extreme sensitivity of the ionization dynamics to the ellipticity of an EP field near CP. Surprisingly, this behavior is reproduced quantitatively by completely classical calculations that, moreover, reveal its cause. In a frame rotating at $\omega$, a CP field at low $\Omega_{0}$ produces a new, slow motion (classically) or a Stark splitting of energy levels (quantally). In the rotating frame an EP field provides $2 \omega$ driving terms that can resonate with this new frequency (splitting). This dramatically influences the ionization dynamics and can be used to control it. It is an excellent example of the kind of manifestly higher-dimensional dynamics that occurs when the polarization of the driving field is no longer restricted to the quasi-1d, LP case.

What about the other dynamical regimes? Will the ionization of excited hydrogen atoms by CP and EP fields be similar to that for the LP field, or will there be new discoveries? Probably yes and yes. As is now being prepared for publication, we have observed $[81,161]$ that at very low $\Omega_{0}$ the quasistatic tunneling of regime-I 
becomes the dominant ionization mechanism even more dramatically for CP than it does for LP. The LP case was studied earlier [1,94].

What about much higher $\Omega_{0}$ ? Will the dynamical localization effect that produces what has been called the quantal suppression of classical chaos cause quantal CP and EP ionization threshold amplitudes to rise systematically above their classical values? The previously predicted [123,124], systematic rise of quantal over classical ionization thresholds was first observed in 1988 for LP driving when $\Omega_{0} \gtrsim 2$ [106]. Another LP hydrogen experiment [129] confirmed this behavior.

Theoretically there is a prediction "that the same phenomenon occurs in the case of elliptically polarized fields" (which includes the CP case); see Sec. VIII of [117] and its bibliography for earlier references on dynamical localization. Coming from authors who helped to initiate the theoretical and numerical study of dynamical localization as an essential phenomenon in physics, this prediction must be tested experimentally. This is a goal for future work in our laboratory.

If the past is any guide, much remains to be discovered, studied, and understood about the polarization dependence of the microwave ionization of excited hydrogen atoms.

\section{Acknowledgments}

The Atomic, Molecular, and Plasma Physics Program of the US National Science Foundation has furnished ongoing support of this research, most recently through grant number PHY-9423001. Michael Nauenberg's persistent but friendly urging to do CP ionization experiments and early financial support by Schlumberger-Doll Research helped the polarization-dependence experiments get underway at Stony Brook. The data reported here were produced as part of the Ph.D. dissertation work of Mark Bellermann at Stony Brook. We mourn the death of our Schlumberger-Doll Research collaborator, colleague, and friend, David Mariani. Derek Richards furnished crucial theoretical support. Steve Zelazny made important recent experimental contributions.

\section{References}

[1] P.M. Koch, K.A.H. van Leeuwen, Phys. Rep. 255, 289 (1995).

[2] P.M. Koch, Physica D 83, 178 (1995).

[3] P.M. Koch, in Chaos/XAOC, Proc. of a Soviet-American Conf., Ed. D.K. Campbell, American Institute of Physics, New York 1990, p. 441.

[4] P.M. Koch, L. Moorman, B.E. Sauer, Comments At. Mol. Phys. 25, 165 (1990).

[5] P.M. Koch, in: Multiphoton Processes, Eds. G. Mainfray, P. Agostini, CEN-CEA Saclay, Gif-sur-Yvette (France) 1990, p. 305.

[6] P.M. Koch, in: Chaos and Quantum Chaos, Ed. W. Dieter Heiss, Lecture Notes in Physics, Vol. 411, Springer-Verlag, Berlin 1992, p. 167.

[7] L. Moorman, in: The Electron, Eds. D. Hestenes, A. Weingartshofer, Kluwer, Dordrecht 1991, p. 353.

[8] L. Moorman, P.M. Koch, in: Quantum Nonintegrability, Eds. D.H. Feng, J.-M. Yuan, World Scientific, Singapore 1992, p. 142.

[9] J.E. Bayfield, S.-Y. Luie, L.C. Perotti, M.P. Skrzypkowski, Physica D 83, 46 (1995). 
[10] R. Blümel, U. Smilansky, J. Opt. Soc. Am. B 7, 664 (1990).

[11] A. Buchleitner, D. Delande, J.-C. Gay, J. Opt. Soc. Am. B 12, 505 (1995).

[12] A. Buchleitner, D. Delande, Chaos Solitons Fractals 5, 1125 (1995).

[13] A. Buchleitner, D. Delande, J. Zakrzewski, in: Multiphoton Processes, Eds. P. Lambropoulos, H. Walther, Institute of Physics, Bristol 1997, p. 19.

[14] G. Casati, I. Guarneri, Comments At. Mol. Phys. 25, 185 (1991).

[15] G. Casati, B.V. Chirikov, Physica D 86, 220 (1995).

[16] G. Casati, B. Chirikov, Quantum Chaos: Between Order and Disorder, Cambridge University Press, Cambridge 1995.

[17] D. Delande, A. Buchleitner, Adv. At. Mol. Opt. Phys. 34, 85 (1994).

[18] R.V. Jensen, Comments At. Mol. Phys. 25, 119 (1990).

[19] R.V. Jensen, S.M. Susskind, M.M. Sanders, Phys. Rep. 201, 1 (1991).

[20] R.V. Jensen, Nature 355, 311 (1992).

[21] J.G. Leopold, D. Richards, J. Phys. B 24, 1209 (1991).

[22] J.G. Leopold, D. Richards, J. Phys. B 27, 2169 (1994).

[23] P.W. Milonni, B. Sundaram, in: Progress in Optics, Vol. XXXI, Ed. E. Wolf, Elsevier, Amsterdam 1993, p. 1.

[24] D. Richards, in: Aspects of Electron-Molecule Scattering and Photoionization, Ed.

A. Herzenberg, American Institute of Physics, New York 1990, p. 55.

[25] D. Richards, J.G. Leopold, in: The Physics of Electronic and Atomic Collisions XVI, Eds. A. Dalgarno, R.S. Freund, P.M. Koch, M.S. Lubell, T.B. Lucatorto, American Institute of Physics, New York 1990, p. 492.

[26] D. Richards, Comments At. Mol. Phys. 25, 195 (1991).

[27] D. Richards, J. Phys. B 25, 1347 (1992).

[28] D. Richards, J. Phys. B 29, 5237 (1996).

[29] D. Richards, J. Phys. B 30, 4019 (1997).

[30] W.E. Baylis, G.W.F. Drake, in: Atomic, Molecular, \& Optical Physics Handbook, Ed. G.W.F. Drake, American Institute of Physics, Woodbury 1996, Ch. 1, p. 1.

[31] J.G. Leopold, I.C. Percival, J. Phys. B 12, 709 (1979).

[32] P.M. Koch, L. Moorman, B.E. Sauer, E.J. Galvez, K.A.H. van Leeuwen, D. Richards, Phys. Scr. Vol. T 26, 51 (1989).

[33] B.E. Sauer, M.R.W. Bellermann, P.M. Koch, Phys. Rev. Lett. 68, 1633 (1992).

[34] Experiment: R.A. Fox, R.M. Kogan, E.J. Robinson, Phys. Rev. Lett. 26, 1416 (1971); theory: P. Lambropoulos, Phys. Rev. Lett. 28, 585 (1972).

[35] L.A. Lompré, G. Mainfray, C. Manus, J. Thébault, Phys. Rev. A 15, 1604 (1977).

[36] Panming Fu, T.J.Scholz, J.M. Hettema, T.F. Gallagher, Phys. Rev. Lett. 64, 511 (1990).

[37] H.R. Reiss, Phys. Rev. Lett. 29, 1129 (1972).

[38] T.F. Gallagher, Mod. Phys. Lett. B 5, 259 (1991).

[39] P. Kappertz, M. Nauenberg, Phys. Rev. A 47, 4749 (1993).

[40] H.R. Reiss, Prog. Quantum Electron. 16, 1 (1992).

[41] C.H. Cheng, C.Y. Lee, T.F. Gallagher, Phys. Rev. A 54, 3303 (1996). 
[42] C.Y. Lee, J.M. Hettema, C.H. Cheng, C.W.S. Conover, T.F. Gallagher, J. Phys. $B$ 29, 3401 (1996).

[43] P.A. Dando, D. Richards, J. Phys. B 26, 3001 (1993).

[44] J. Mostowski, J.J. Sanchez-Mondragon, Opt. Commun. 29, 293 (1979).

[45] B.I. Meerson, Opt. Spektrosk. 51, 582 (1981) [Opt. Spectrosc. (USSR) 51, 322 (1981)].

[46] N.B. Delone, B.P. Krainov, D.L. Shepelyansky, Usp. Fiz. Nauk 140, 355 (1983) [Sov. Phys. Usp. 26, 551 (1983)].

[47] I. Białynicki-Birula, M. Kaliński, J.H. Eberly, Phys. Rev. Lett. 73, 1777 (1994). See Comment by D. Farrelly, E. Lee, T. Uzer, Phys. Rev. Lett. 75, 972 (1995) and Reply, loc. cit., p. 973.

[48] I. Białynicki-Birula, M. Kaliński, J.H. Eberly, Phys. Rev. Lett. 75, 973 (1995).

[49] A. Buchleitner, D. Delande, Phys. Rev. Lett. 75, 1487 (1995).

[50] A.F. Brunello, T. Uzer, D. Farrelly, Phys. Rev. Lett: 76, 2874 (1996).

[51] A.F. Brunello, T. Uzer, D. Farrelly, Phys. Rev. A 55, 3730 (1997).

[52] D. Delande, R. Gębarowski, M. Kuklińska, B. Piraux, K. Rzążewski, J. Zakrzewski, in: Super-Intense Laser-Atom Physics, Eds. B. Piraux, A. L'Huillier, K. Rzążewski, Plenum Press, New York 1993, p. 317.

[53] D. Farrelly, T. Uzer, Phys. Rev. Lett. 74, 1720 (1995).

[54] D. Farrelly, E. Lee, T. Uzer, Phys. Lett. A 204, 359 (1995).

[55] D. Farrelly, E. Lee, T. Uzer, Phys. Rev. Lett. 75, 972 (1995), a Comment, for which the Reply is Phys. Rev. Lett. 75, 973 (1995).

[56] D. Farrelly, Phys. Rev. A 55, 1550 (1997).

[57] M. Gajda, B. Piraux, K. Rzążewski, Phys. Rev. A 50, 2528 (1994).

[58] K. Ganesan, R. Gębarowski, Pramana 48, 379 (1997).

[59] R. Gębarowski, J. Zakrzewski, Phys. Rev. A 51, 1508 (1995).

[60] J.A. Griffiths, D. Farrelly, Phys. Rev. A 45, R2678 (1992).

[61] T.P. Grozdanov, M.J. Raković, E.A. Solov'ev, J. Phys. B 25, 4455 (1992).

[62] J.E. Howard, Phys. Rev. A 46, 364 (1992).

[63] J.E. Howard, Phys. Rev. A 51, 3934 (1995). See Comment on this article by D. Farrelly, Phys. Rev. A 55, 1550 (1997) and the Reply by J. Howard, ibid. 55, 1552 (1997).

[64] M. Kalinski, J.H. Eberly, I. Bialynicki-Birula, Phys. Rev. A 52, 2460 (1995).

[65] M. Kalinski, J.H. Eberly, Phys. Rev. A 53, 1715 (1996).

[66] M. Kalinski, J.H. Eberly, Phys. Rev. Lett. 77, 2420 (1996).

[67] H. Klar, Z. Phys. D 11, 45 (1989).

[68] M. Nauenberg, Phys. Rev. Lett. 64, 2731 (1990).

[69] A. Peregrine-Smew, D. Farrelly, T. Uzer, Phys. Rev. A 51, 4293 (1995).

[70] M.J. Raković, S.-I. Chu, Phys. Rev. A 50, 5077 (1994).

[71] M.J. Raković, S.-I. Chu, Physica D 81, 271 (1995).

[72] M.J. Raković, S.-I. Chu, Phys. Rev. A 75, 1358 (1995).

[73] K. Rzążewski, B. Piraux, Phys. Rev. A 47, R1612 (1993). 
[74] K. Sacha, J. Zakrzewski, Phys. Rev. A 55, 568 (1997).

[75] D. Wintgen, Z. Phys. D 18, 125 (1991).

[76] J. Zakrzewski, D. Delande, J.-C. Gay, K. Rzążewski, Phys. Rev. A 47, R2468 (1993).

[77] J. Zakrzewski, D. Delande, J. Phys. B 28, L667 (1995).

[78] J. Zakrzewski, R. Gębarowski, D. Delande, Phys. Rev. A 54, 691 (1996).

[79] J. Zakrzewski, D. Delande, J. Phys. B 30, L87 (1997).

[80] P.M. Koch, in: Rydberg States of Atoms and Molecules, Eds. R.F. Stebbings, F.B. Dunning, Cambridge University Press, London 1983, p. 473.

[81] M.R.W. Bellermann, Ph.D. thesis, State University of New York at Stony Brook, Physics Department, 1995.

[82] M.R.W. Bellermann, P.M. Koch, D.R. Mariani, D. Richards, Phys. Rev. Lett. 76, 892 (1996).

[83] M.R.W. Bellermann, P.M. Koch, D. Richards, Phys. Rev. Lett. 78, 3840 (1997).

[84] B. Sauer, K.A.H. van Leeuwen, A. Mortazawi-M., P.M. Koch, Rev. Sci. Instrum. 62, 189 (1991).

[85] S.A. Zelazny, P.M. Koch, Bull. Am. Phys. Soc. 42, 932 (1997).

[86] S. Yoakum, L. Sirko, P.M. Koch, Phys. Rev. Lett. 69, 1919 (1992).

[87] L. Sirko, M.R.W. Bellermann, A. Haffmans, P.M. Koch, D. Richards, Phys. Rev. Lett. 71, 2895 (1993).

[88] A. Haffmans, R. Blümel, P.M. Koch, L. Sirko, Phys. Rev. Lett. 73, 248 (1994).

[89] L. Sirko, A. Haffmans, M.R.W. Bellermann, P.M. Koch, Europhys. Lett. 33, 181 (1996).

[90] J.E. Bayfield, L.D. Gardner, P.M. Koch, Phys. Rev. Lett. 39, 76 (1977).

[91] O. Rath, Ph.D. thesis, The Open University, Mathematics Faculty, 1990.

[92] O. Rath, D. Richards, 1997, to be submitted to J. Phys. B.

[93] D. Richards, in: Classical, Semiclassical, and Quantum Dynamics in Atoms, Eds.

H. Friedrich, B. Eckhardt, Springer, Berlin 1997.

[94] B.E. Saver, S. Yoakum, L. Moorman, P.M. Koch, D. Richards, P.A. Dando, Phys. Rєv. Lett. 68, 468 (1992).

[95] D. Richards, J. Phys. B 20, 2171 (1987).

[96] D. Richards, J.G. Leopold, P.M. Koch, E.J. Galvez, K.A.H. van Leeuwen, L. Moorman, B.E. Sauer, R.V. Jensen, J. Phys. B 22, 1307 (1989).

[97] R. Blümel, U. Smilansky, Phys. Rev. Lett. 58, 2531 (1987).

[98] H.P. Breuer, M. Holthaus, Z. Phys. D 11, 1 (1989).

[99] G.P. Berman, G.M. Zaslavsky, Phys. Lett. A 61, 295 (1977).

[100] B.V. Chirikov, Phys. Rep. 52, 263 (1979).

[101] B.I. Meerson, E.A. Oks, P.V. Sasorov, Pis'ma Zh. Eksp. Teor. Fiz. 29, 79 (1979) [Sov. Phys.-JETP Lett. 29, 72 (1979)].

[102] G.M. Zaslavsky, Phys. Rep. 80, 157 (1981).

[103] A.J. Lichtenberg, M.A. Lieberman, Regular and Stochastic Motion, Springer, New York 1983.

[104] J.D. Meiss, Rev. Mod. Phys. 64, 795 (1992). 
[105] L. Sirko, P.M. Koch, Appl. Phys. B 60, S195 (1995).

[106] E.J. Galvez, B.E. Sauer, L. Moorman, P.M. Koch, D. Richards, Phys. Rev. Lett. 61, 2011 (1988).

[107] P.M. Koch, Chaos 2: 131 (1992).

[108] R.V. Jensen, S.M. Susskind, M.M. Sanders, Phys. Rev. Lett. 62, 1476 (1989).

[109] R.V. Jensen, M.M. Sanders, M. Saraceno, B. Sundaram, Phys. Rev. Lett. 63, 2771 (1989).

[110] H.P. Breuer, K. Dietz, M. Holthaus, Z. Phys. D 18, 239 (1991).

[111] R.V. Jensen, in: Quantum Dynamics of Chaotic Systems, Eds. J.-M. Yuan, D.H. Feng, G.M. Zaslavsky, Gordon and Breach, Langhorne (PA) 1993, p. 113.

[112] G. Radons, R.E. Prange, Phys. Rev. Lett. 61, 1691 (1988).

[113] G. Radons, R.E. Prange, in: Quantum Chaos, Proc. Adriatico Research Conf. and Miniworkshop, 1990, Eds. H.A. Cerdeira, R. Ramaswamy, M.C. Gutzwiller, World Scientific, Singapore 1991, p. 333.

[114] R.E. Prange, in: Chaos and Quantum Chaos, Ed. W. Dieter Heiss, Lecture Notes in Physics, Vol. 411, Springer-Verlag, Berlin 1992, p. 225.

[115] G. Casati, B.V. Chirikov, I. Guarneri, D.L. Shepelyansky, Phys. Rev. Lett. 59, 2927 (1987).

[116] G. Casati, I. Guarneri, D.L. Shepelyansky, Phys. Rev. A 36, 3701 (1987).

[117] G. Casati, I. Guarneri, D.L. Shepelyansky, IEEE J. Quantum Electron. 24, 1420 (1988).

[118] K. Wang, S.-I. Chu, Phys. Rev. A 39, 1800 (1989).

[119] A. Buchleitner, D. Delande, Phys. Rev. Lett. 70, 33 (1993).

[120] A. Buchleitner, D. Delande, Phys. Rev. Lett. 71, 3633 (1993).

[121] A. Buchleitner, B. Grémaud, D. Delande, J. Phys. B 27, 2663 (1994).

[122] A. Buchleitner, D. Delande, Phys. Rev. A 55, R1585 (1997).

[123] D.L. Shepelyansky, Physica D 8, 208 (1983).

[124] G. Casati, B.V. Chirikov, D.L. Shepelyansky, Phys. Rev. Lett. 53, 2525 (1984).

[125] G. Casati, B.V. Chirikov, D.L. Shepelyansky, I. Guarneri, Phys. Rep. 154, 77 (1987).

[126] G. Casati, L. Molinari, Prog. Theor. Phys. Suppl. 98, 287 (1989).

[127] B.V. Chirikov, in: Chaos and Quantum Physics, Eds. M.-J. Giannoni, A. Voros, J. Zinn-Justin, Elsevier, Amsterdam 1991, p. 444.

[128] G. Casati, B. Chirikov, Quantum Chaos: Between Order and Disorder, Cambridge University Press, Cambridge 1995, p. 3.

[129] J.E. Bayfield, G. Casati, I. Guarneri, D.W. Sokol, Phys. Rev. Lett. 63, 364 (1989).

[130] M. Arndt, A. Buchleitner, R.N. Mantegna, H. Walther, Phys. Rev. Lett. 67, 2435 (1991).

[131] S. Fishman, D.R. Grempel, R.E. Prange, Phys. Rev. Lett. 49, 509 (1982).

[132] D.R. Grempel, R.E. Prange, S. Fishman, Phys. Rev. A 29, 1639 (1984).

[133] F.L. Moore, J.C. Robinson, C. Bharucha, P.E. Williams, M.G. Raizen, Phys. Rev. Lett. 73, 2974 (1994). 
[134] F.L. Moore, J.C. Robinson, C.F. Bharucha, B. Sundaram, M.G. Raizen, Phys. Rev. Lett. 75, 4598 (1995).

[135] J.C. Robinson, C. Bharucha, F.L. Moore, R. Jahnke, G.A. Georgakis, Q. Niu, M.G. Raizen, B. Sundaram, Phys. Rev. Lett. 74, 3963 (1995).

[136] J.G. Leopold, D. Richards, Phys. Rev. A 38, 2660 (1988).

[137] R.V. Jensen, J.G. Leopold, D. Richards, J. Phys. B 21, L527 (1988).

[138] D. Richards, J.G. Leopold, R.V. Jensen, J. Phys. B 22, 417 (1988).

[139] J.G. Leopold, D. Richards, J. Phys. B 22, 1931 (1989).

[140] R.S. MacKay, J.D. Meiss, Phys. Rev. A 37, 4702 (1988).

[141] J.D. Meiss, Phys. Rev. Lett. 62, 1576 (1989).

[142] See the papers in Atoms in Intense Laser Fields, Ed. M. Gavrila, Adv. Atom. Mol. Opt. Phys. Supplement 1, Academic, San Diego 1992 and in the different proceedings whose titles follow that of the conference series Super-Intense Laser-Atom Physics, Eds. B. Piraux, A. L'Huillier, K. Rzążewski, Plenum Press, New York 1993; Eds. H.G. Muller, M.V. Fedorov, Kluwer, Dordrecht 1996.

[143] M.P. de Boer, J.H. Hoogenraad, R.B. Vrijen, L.D. Noordham, H.G. Muller, Phys. Rev. Lett. 71, 3263 (1993).

[144] M.P. de Boer, J.H. Hoogenraad, R.B. Vrijen, R.C. Constantinescu, L.D. Noordham, H.G. Muller, Phys. Rev. A 50, 4085 (1994).

[145] (a) V. Gontis, B. Kaulakys, J. Phys. B 20, 5051 (1987); (b) G. Casati, I. Guarneri, D.L. Shepelyansky, IEEE J. Quantum Electron. 24, 1420 (1988).

[146] R. Blümel, Phys. Rev. A 49, 4787 (1994).

[147] J.E. Bayfield, S.Y. Luie, L.C. Perotti, M.P. Skrzypkowski, Phys. Rev. A 53, R12 (1996).

[148] V.I. Arnold, Mathematical Methods of Classical Mechanics, Springer-Verlag, New York 1978.

[149] L.E. Reichl, The Transition to Chaos: In Conservative Classical Systems: Quantum Manifestations, Springer, New York 1992.

[150] B. Sundaram, R.V. Jensen, Phys. Rev. A 51, 4018 (1995).

[151] D. Banks, J.G. Leopold, J. Phys. B 11, 2833 (1978).

[152] P.M. Koch, D.R. Mariani, Phys. Rev. Lett. 46, 1275 (1981).

[153] LP: A. Buchleitner, D. Delande, Phys. Rev. Lett. 75, 1487 (1995); CP: D. Delande, J. Zakrzewski, A. Buchleitner, Europhys. Lett. 32, 107 (1995).

[154] G.P. Brivio, G. Casati, L. Perotti, I. Guarneri, Physica D 33, 51 (1988).

[155] D. Richards, in preparation.

[156] J.G. Leopold, D. Richards, J. Phys. B 19, 1125 (1986).

[157] R.V. Jensen, Phys. Scr. 35, 668 (1987).

[158] K. Sacha, J. Zakrzewski, Phys. Rev. A 56, 719 (1997).

[159] J. Henkel, M. Holthaus, Phys. Rev. A 45, 1978 (1992).

[160] M.J. Davis, E.J. Heller, J. Chem. Phys. 75, 246 (1981).

[161] M.R.W. Bellermann, P.M. Koch, D. Richards, Bull. Am. Phys. Soc. 41, 1092 (1996). 